Global solvability of systems on compact surfaces

Giuliano Angelo Zugliani 

SERVIÇO DE PÓS-GRADUAÇÃO DO ICMC-USP

Data de Depósito:

Assinatura:

\title{
Global solvability of systems on compact surfaces
}

\section{Giuliano Angelo Zugliani}

\author{
Advisor: Prof. Dr. Adalberto Panobianco Bergamasco \\ Co-advisor: Prof. Dr. Sérgio Luís Zani \\ Co-advisor: Prof. Dr. Alberto Parmeggiani
}

Doctoral dissertation submitted to the Instituto de Ciências Matemáticas e de Computação - ICMC-USP, in partial fulfillment of the requirements for the degree of the Doctorate Program in Mathematics. FINAL VERSION. 
Ficha Catalográfica elaborada pela Seção de Tratamento da Informação da Biblioteca Prof. Achille Bassi- Instituto de Ciências Matemáticas e de Computação - ICMC/USP.

\section{Zugliani, Giuliano Angelo}

Z94g Global sovability of systems on compact surfaces / Giuliano Angelo

Zugliani; thesis advisor Adalberto Panobianco Bergamasco; joint supervisor Sérgio Luis

Zani and Alberto Parmeggiani. - - São Carlos, 2014.

$61 \mathrm{p}$.

Tese (Doutorado) - Programa de Pós-Graduação em Ciências de Computação e

Matemática Computacional . - - Instituto de Ciências Matemáticas e de Computação, Universidade de São Paulo, 2014.

1. Complex vector fields. 2.Global solvability. 3. Involutive systems.

I. Bergamasco, Adalberto Panobianco, thesis advisor. II. Zani, Sérgio Luis, joint

III. supervisor. III. Parmeggiani, Alberto, joint supervisor. IV. Títle 
Resolubilidade global de sistemas em superfícies compactas

Giuliano Angelo Zugliani 



\title{
Resolubilidade global de sistemas em superfícies compactas
}

\author{
Giuliano Angelo Zugliani
}

\author{
Orientador: Prof. Dr. Adalberto Panobianco Bergamasco \\ Coorientador: Prof. Dr. Sérgio Luís Zani \\ Coorientador: Prof. Dr. Alberto Parmeggiani
}

Tese apresentada ao Instituto de Ciências Matemáticas e de Computação - ICMC-USP, como parte dos requisitos para obtenção do título de Doutor em Ciências - Matemática. VERSÃO REVISADA. 
Ficha Catalográfica elaborada pela Seção de Tratamento da Informação da Biblioteca Prof. Achille Bassi- Instituto de Ciências Matemáticas e de Computação - ICMC/USP.

Zugliani, Giuliano Angelo

Z94r Resolubilidade global de sistemas em superfícies compactas / Giuliano Angelo

Zugliani; orientador Adalberto Panobianco Bergamasco; co-orientador Sérgio Luis Zani e

Alberto Parmeggiani. - - São Carlos, 2014.

$61 \mathrm{p}$.

Tese (Doutorado) - Programa de Pós-Graduação em Ciências de Computação e

Matemática Computacional . - - Instituto de Ciências Matemáticas e de Computação,

Universidade de São Paulo, 2014.

1. Campos vetoriais complexos. 2.Resolubilidade global. 3. Sistemas involutivos.

I. Bergamasco, Adalberto Panobianco, orient. II. Zani, Sérgio Luis, co-orient. III.

Parmeggiani, Alberto, co-orient. IV. Título. 


\section{AGRADECIMENTOS}

Concluir uma etapa é sempre motivo de alegria. Concluir uma etapa que você descobriu ser mesmo seu objetivo é um motivo de satisfação maior ainda. Uma jornada menos natural e seus obstáculos podem valorizar mais a conquista.

Tenho muito a agradecer a Deus nesta jornada. Dedico este trabalho a todos os meus familiares, em especial a minha querida Rosana (que é de fato a minha família agora) e aos meus pais e avós, cujo apoio foi imprescindível.

Aos meus amigos de doutorado, muito obrigado pelos momentos de estudo e de atividades esportivas. Aos meus colegas de trabalho, muito obrigado pela amizade e pelo que me ensinaram.

Ao Prof. Adalberto, que sempre me foi um exemplo de ensino e motivação, agradeço pela orientação e por ter me confiado este belo problema, desde a tarde de 11 de outubro de 2010.

Ao Prof. Zani, muito obrigado pela coorientação, pela dedicação nos seminários com o Prof. Adalberto e pela tese do Levitt.

Neste trabalho foi fundamental o estágio no exterior apoiado pela FAPESP. Agradeço ao Prof. Alberto pela coorientação e pela supervisão nesta visita. A Università di Bologna e seus alunos me recepcionaram muito bem e me proporcionaram uma ótima experiência.

Espero neste trabalho ter correspondido minimamente ao padrão de pesquisa destes professores. Considero nosso encontro em Serra Negra fundamental para o projeto.

Ao Prof. Paulo Cordaro, que viu em Pisa, há um ano, muitas destas ideias nascendo, meu obrigado pela atenção e pelo incentivo.

Agradeço à FAPESP e ao CNPq pelo suporte durante o doutorado no Brasil.

Finalmente, dedico esta tese também a todos os que foram meus professores, desde os níveis mais básicos. 


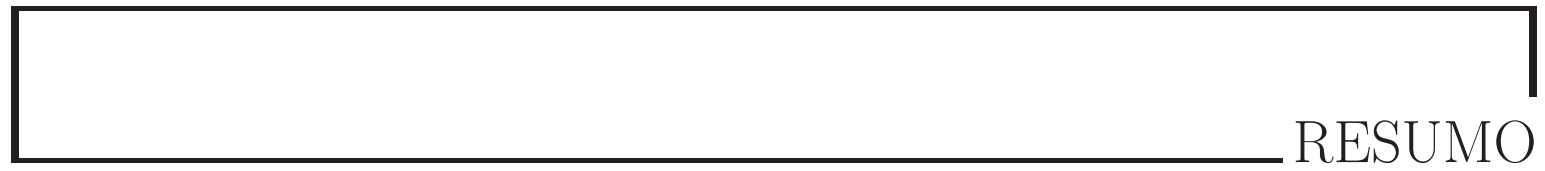

Nosso interesse é estudar um sistema involutivo definido por uma 1-forma fechada e não-exata em uma superfície fechada e orientável.

Apresentamos aqui uma condição necessária para a resolubilidade global desde sistema. Nós também construímos exemplos de sistemas globalmente resolúveis que nos permitiram fornecer a equivalência entre a resolubilidade global e a condição necessária, para dois casos envolvendo 1-formas do tipo Morse: quando a superfície é o bitoro ou quando a 1-forma é genérica. 


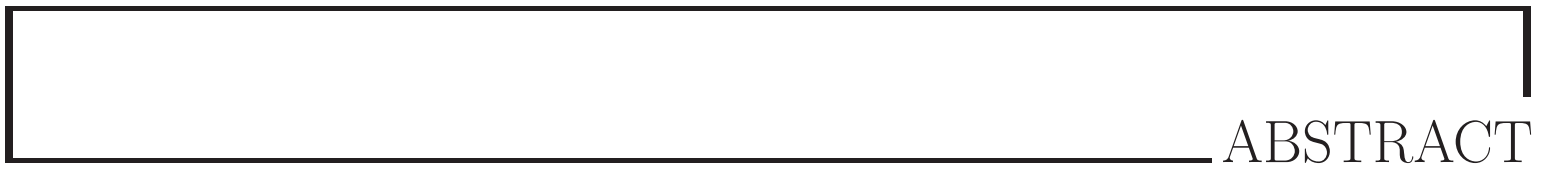

We are interested in studying an involutive system defined by a closed non-exact 1-form on a closed and orientable surface.

Here we present a necessary condition for the global solvability of this system. We also make some particular constructions of globally solvable systems that motivate the equivalence between the global solvability and the necessary condition, for two cases involving 1-forms of the Morse type, namely, when the surface is the bitorus or when the 1 -form is generic. 
Let $b$ be a real smooth closed non-exact 1-form defined on a closed orientable surface $M$.

We consider the operator $\mathbb{L}: \mathscr{D}^{\prime}\left(M \times \mathbb{S}^{1}\right) \rightarrow \bigwedge^{1} \mathscr{D}^{\prime}\left(M \times \mathbb{S}^{1}\right)$ defined by

$$
\mathbb{L} u=d_{t} u+i b(t) \wedge \partial_{x} u .
$$

Our aim is to find necessary conditions and sufficient conditions for the global solvability of $\mathbb{L}$.

This operator defines a differential complex

$$
0 \longrightarrow \mathscr{D}^{\prime}\left(M \times \mathbb{S}^{1}\right) \stackrel{\mathbb{L}}{\longrightarrow} \bigwedge^{1} \mathscr{D}^{\prime}\left(M \times \mathbb{S}^{1}\right) \stackrel{\mathbb{L}^{1}}{\longrightarrow} \bigwedge^{2} \mathscr{D}^{\prime}\left(M \times \mathbb{S}^{1}\right) \stackrel{\mathbb{L}^{2}}{\longrightarrow} 0
$$

and, since $b$ is closed, we have $\mathbb{L}^{1} \circ \mathbb{L}=0$. Because of this, if we want to solve $\mathbb{L} u=f$, we must have $\mathbb{L}^{1} f=0$, which are compatibility conditions related to the complex. This let us alert to look for more compatibility conditions, related to the nature of $M$.

First we briefly discuss the history of the problem.

In [Tr2], it is proved that the semiglobal solvability (in the context of pseudodifferential operators) on any manifold is related to the property of the sublevels and the superlevels of a local primitive of $b$ being connected.

In the case when $b$ is exact, in $[\mathrm{CH}]$ the authors studied the problem for a closed and orientable manifold $M$ (in the context of linear self-adjoint operators in a Hilbert space). A primitive $B(t)=\int_{t_{0}}^{t} b$ is defined on $M$, and we have

Theorem $(\mathrm{CH})$. If $b$ is exact, then $\mathbb{L}$ is globally solvable if and only if $\{t \in M: B(t)<$ $r\}$ and $\{t \in M: B(t)>r\}$ are connected, for every $r \in \mathbb{R}$.

When $b$ is closed, but not exact, and $M$ is the torus $\mathbb{T}^{2}$ the authors in $[\mathrm{BK}]$ obtained a result by using the universal covering space $\mathbb{R}^{2}$ on which $B$ is defined, when the rank over $\mathbb{Q}$ of the following subgroup of $\mathbb{R}$, 


$$
\operatorname{Per}(b)=\left\{\int_{\gamma} b: \gamma \text { is a loop in } \mathbb{T}^{2}\right\}
$$

is maximal, namely 2. The proof of this case was completed in [BKNZ]. There, it was important to view a primitive $B$ as defined on an intermediate covering space (the cylinder), when the periods are commensurable, that is, linearly dependent over $\mathbb{Q}$, and we may state

Theorem (BK,BKNZ). $\mathbb{L}$ is globally solvable if and only if $\left\{t \in \mathbb{R}^{2}: B(t)<r\right\}$ and $\left\{t \in \mathbb{R}^{2}: B(t)>r\right\}$ are connected, for every $r \in \mathbb{R}$.

The compatibility conditions for $\mathbb{L} u=f$ to have a solution $u \in \mathscr{D}^{\prime}\left(\mathbb{T}^{3}\right)$, if $f \in \bigwedge^{1} C^{\infty}\left(\mathbb{T}^{3}\right)$, are

$$
\mathbb{L}^{1} f=0 \text { and } \int_{0}^{2 \pi} \int_{0}^{2 \pi} f_{1}\left(t_{1}, 0, x\right) d t_{1} d x=\int_{0}^{2 \pi} \int_{0}^{2 \pi} f_{2}\left(0, t_{2}, x\right) d t_{2} d x=0
$$

where $(t, x)=\left(t_{1}, t_{2}, x\right)$ are the coordinates in $\mathbb{T}^{3}$ and $f(t, x)=f_{1}(t, x) d t_{1}+f_{2}(t, x) d t_{2}$.

We organize this work as follows.

Chapter 1 is dedicated to the background and to defining the compatibility conditions for our system. Constructing a minimal covering $\widetilde{M}$ of $M$ on which there is a pseudoperiodic primitive of $b$ is crucial for this. In Chapter 2, we prove that a certain condition about the sublevels and the superlevels of this primitive in $\widetilde{M}$ implies that the system is not globally solvable; such a condition is no longer the non-connectedness of a sublevel (or a superlevel), but rather the boundedness of $B$ on a component of a sublevel (or a superlevel). The heart of the proof consists of violating a priori estimates, as in [Tr2], and it enhances the results about the necessary condition in [BK, BKNZ], on which the authors work with the method of stationary phase. Nonetheless, at the end of Chapter 2, we present another proof, which reduces the problem to the torus when this component is indeed bounded.

With regards to a sufficient condition, we dedicate Chapters 3 through 5 . The inspiration comes from [Tr2, BK, BKNZ], but here we do not make use of global coordinates. In order to head off this difficulty, we work with some instructive constructions of forms given by [Far], which have inspired us to furnish a class of globally solvable systems.

Also, this lead us to consider Morse 1-forms: thanks to a theorem by Levitt, in Chapter 4 we prove the global solvability for any genus if $b$ is a generic Morse form. In Chapter 5, we complete the cases for Morse forms on the bitorus obtaining an equivalence between our condition and the solvability. The final chapter, in turn, 
yields the equivalence for a special case on which $b$ has rank equal to 1 and supplies more examples.

Working with Morse forms is far from being particular: the set of Morse forms is open and dense in the set of the smooth closed 1-forms. All the machinery involved in this part enables us to consider, in the future, the problem when $b$ is a real analytic 1-form, for instance, as we can see in Example 5.5. 
1 Introduction 1

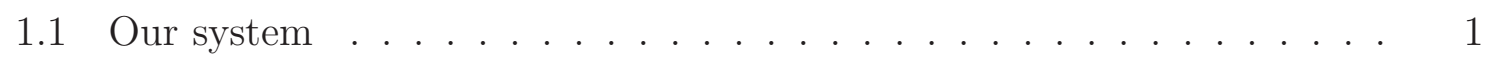

1.2 Minimal covering spaces . . . . . . . . . . . . . . 4

1.3 Examples ......................... 6

1.4 Compatibility conditions . . . . . . . . . . . . . 7

2 A necessary condition for solvability 11

2.1 Auxiliary lemmas . . . . . . . . . . . . . . . . . . . . 13

2.2 Construction of the forms and proof . . . . . . . . . . . . . . . . . 14

2.3 Another proof . . . . . . . . . . . . . . . . 17

$\begin{array}{lll}3 & \text { Examples of globally solvable systems } & 19\end{array}$

3.1 Foliations . . . . . . . . . . . . . . . . . . . 19

3.2 Construction of 1 -forms . . . . . . . . . . . . . . . . 22

3.2 .1 Case A . . . . . . . . . . . . . . . 23

3.2 .2 Case B . . . . . . . . . . . . . . . . 24

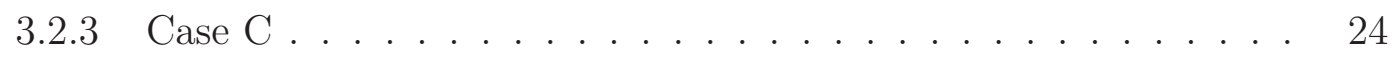

3.2.4 Case D . . . . . . . . . . . . . . . . 24

3.3 Our class of examples and auxiliary results . . . . . . . . . . . 25

3.3.1 Incommensurable case . . . . . . . . . . . . . 27

3.3 .2 Lower rank . . . . . . . . . . . . . . . . . . . . . . 29

3.4 Proof of the global solvability . . . . . . . . . . . . . . . . 31

3.5 Morse forms . . . . . . . . . . . . . . . . . . . . . . 34

4 Global solvability for the generic case $\quad 37$

4.1 Construction of almost transversal curves . . . . . . . . . . . . . . . . . 37

4.2 Proof of the generic case . . . . . . . . . . . . . . . . . 40 
5 Bitorus revisited $\quad 45$

5.1 Case $1 \ldots \ldots \ldots \ldots \ldots \ldots$

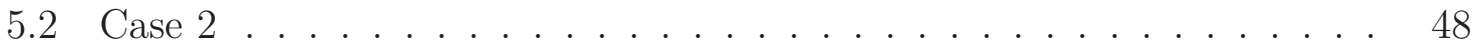

5.3 Construction of an almost transversal curve . . . . . . . . . . . . . . . 49

6 Further examples $\quad 55$

6.1 A case with rank $1 \ldots \ldots \ldots \ldots 5$

6.2 Non-solvability revisited . . . . . . . . . . . . . . . 57 
1.1 Cutting where the periods are zero . . . . . . . . . . . . 8

2.1 Construction of $\gamma \ldots \ldots \ldots \ldots \ldots$

2.2 Support of $d_{t} v_{0} \ldots \ldots \ldots \ldots \ldots \ldots$

3.1 Case A . . . . . . . . . . . . . . . . . . . 23

3.2 Case B . . . . . . . . . . . . . . . . . . . . . 24

3.3 Case C . . . . . . . . . . . . . . . . . . . . . 24

3.4 Case D . . . . . . . . . . . . . . . . . . . 25

3.5 Higher genus . . . . . . . . . . . . . . . . . . . . 26

3.6 Surfaces with pipes attached . . . . . . . . . . . . . . 26

3.7 Position B and $\omega_{1}=d \theta \ldots \ldots \ldots$. . . . . . . . . . . 30

3.8 Position $\mathrm{C}$ and $\omega_{1}=d \theta \ldots \ldots \ldots \ldots \ldots \ldots$

4.1 Decomposition in pants . . . . . . . . . . . . . . . 38

4.2 The paths $\alpha_{1}$ and $\alpha_{2} \ldots \ldots \ldots \ldots \ldots$

4.3 Construction of $\alpha$ for genus $2 \ldots \ldots \ldots$. . . . . . . . . . . 40

4.4 Almost transversal curves for genus greater than 2 . . . . . . . . . . 40

4.5 Cut avoiding the boundary components . . . . . . . . . . . . . . . . 42

5.1 Case $1.1 \ldots \ldots \ldots \ldots$

5.2 Case $1.2 \ldots \ldots \ldots \ldots \ldots$. . . . . . . . . . . . . . . . . . . . . . . . 47

5.3 Case $1.3 \ldots \ldots \ldots \ldots$. . . . . . . . . . . . . . . . . . . . . 48

5.4 A singular level crossing boundary components . . . . . . . . . . . . . . 51

5.5 Saddles connected by a leaf in $M_{1} \ldots \ldots$. . . . . . . . . . . . 52

5.6 An example having only one compactifiable arc . . . . . . . . . . . 53

5.7 A real analytic example . . . . . . . . . . . . . . . . 54

6.1 Covering space for rank $1 \ldots \ldots \ldots \ldots 5$ 


\section{CHAPTER 1}

INTRODUCTION

La topologie est précisément la discipline mathématique qui permet la passage du local au global.

- R. Thom.

\subsection{Our system}

We shall assume some familiarity with terminology and basic facts about homology and differential forms. Here we recall some of them and fix the notations.

Define the linear operator $\mathbb{L}: \mathscr{D}^{\prime}\left(M \times \mathbb{S}^{1}\right) \rightarrow \bigwedge^{1} \mathscr{D}^{\prime}\left(M \times \mathbb{S}^{1}\right)$ by

$$
\mathbb{L} u=d_{t} u+i b(t) \wedge \partial_{x} u,
$$

where $b$ is a real smooth closed 1-form defined on a closed (compact without boundary) and orientable surface $M$. The symbol $\partial_{x}$ denotes the derivative with respect to $x$ belonging to the circle $\mathbb{S}^{1}$, and $d_{t}: \bigwedge^{j} \mathscr{D}^{\prime}(M) \rightarrow \bigwedge^{j+1} \mathscr{D}^{\prime}(M)$ is the exterior derivative on $M, j=0,1$.

This operator can be inserted in the context of $[\mathrm{BCH}]$ : let $T^{\prime}$ be the line subbundle of $\mathbb{C} \otimes T^{*}\left(M \times \mathbb{S}^{1}\right)$ generated by $d x-i b$, which is a locally integrable structure called a tube structure. Then, $\mathscr{V} \doteq\left(T^{\prime}\right)^{\perp}$ is locally generated by the vector fields

$$
L_{j}=\frac{\partial}{\partial t_{j}}+i \frac{\partial B}{\partial t_{j}}(t) \frac{\partial}{\partial x}, \quad j=1,2,
$$

where $\left(t_{1}, t_{2}\right)$ are local coordinates on $M$ and $B$ is a local primitive of $b$, which means that locally we have a system of linear first order partial differential equations. We will write $\frac{\partial}{\partial t_{j}}$ shortly as $\partial_{j}$, with $j=1,2$. 
Furthermore, the computation $\mathbb{L}^{1}(\mathbb{L} u)=\mathbb{L}^{1}\left(L_{1} u d t_{1}+L_{2} u d t_{2}\right)=\left[L_{2}, L_{1}\right] u d t_{1} \wedge$ $d t_{2}=0$ reveals that $\mathscr{V}$ is a formally integrable structure.

Concerning the characteristic set, $\eta \in T_{(t, x)}^{0}$ is non-trivial if and only if $\frac{\partial B}{\partial t_{1}}(t)=$ $\frac{\partial B}{\partial t_{2}}(t)=0$ and $\eta=\eta_{x} d x$, with $\eta_{x} \in \mathbb{R} \backslash\{0\}$.

The Levi form of a formally integrable structure at $\eta \in T_{(t, x)}^{0}, \eta \neq 0$, is the hermitian form given by

$$
\mathcal{L}(L, M)=\frac{1}{2 i} \eta([L, \bar{M}]) .
$$

Therefore, in our case, if $L=v_{1} L_{1}+v_{2} L_{2}$ and $M=w_{1} L_{1}+w_{2} L_{2}$, with $v_{j}, w_{j} \in \mathbb{C}$ for $j=1,2$, we have

$$
\begin{aligned}
& \mathcal{L}(L, M)=\frac{1}{2 i} \eta\left(\sum_{j, k=1}^{2} v_{j} \overline{w_{k}}\left[L_{j}, \overline{L_{k}}\right]\right)= \\
& =\frac{1}{2 i} \eta\left(\sum_{j, k=1}^{2} v_{j} \overline{w_{k}}(-2 i) \frac{\partial^{2} B}{\partial t_{j} \partial t_{k}}(t) \partial x\right)=-\eta_{x}\left(\begin{array}{ll}
v_{1} & v_{2}
\end{array}\right) \operatorname{Hess}_{t} B\left(\overline{w_{1}} \quad \overline{w_{2}}\right)^{t} .
\end{aligned}
$$

In some chapters, we will consider systems defined by Morse forms, which, by the above computation, is equivalent to requiring that the Levi form is non-degenerate at any $\eta \in T_{(t, x)}^{0}, \eta \neq 0$. A non-elliptic formally integrable structure of codimension 1 satisfying this is called a Mizohata structure.

We will be interested in finding a global solution to the introduced system; by this we mean a $u \in \mathscr{D}^{\prime}\left(M \times \mathbb{S}^{1}\right)$ such that $\mathbb{L} u=f \in \bigwedge^{1} C^{\infty}\left(M \times \mathbb{S}^{1}\right)$. The solutions found here will be indeed smooth.

The main technique used in order to attain this is to study the decay of the Fourier coefficients of the expansions

$$
u(t, x)=\sum_{\xi \in \mathbb{Z}} \hat{u}(t, \xi) e^{i x \xi} \text { and } f(t, x)=\sum_{\xi \in \mathbb{Z}} \hat{f}(t, \xi) e^{i x \xi}
$$

with respect to $x \in \mathbb{S}^{1}$. A candidate to a solution should satisfy, for every $\xi \in \mathbb{Z}$, the differential equation

$$
d_{t} \hat{u}(t, \xi)-\xi b(t) \hat{u}(t, \xi)=\hat{f}(t, \xi) .
$$

We have, for every $N \in \mathbb{Z}^{+}$, a constant $C_{N}>0$ such that

$$
|\hat{f}(t, \xi)| \leqslant \frac{C_{N}}{(1+|\xi|)^{N}}
$$

and we will be looking for a similar rapid decay for $\{\hat{u}(t, \xi)\}_{\xi \in \mathbb{Z} \text {. }}$ 
Next, let us inspect more closely the elements involved in our system.

Every orientable surface $M$ is diffeomorphic to a connected sum of $g$ tori. The number $g$ is said to be the genus of the surface.

By using the intersection pairing of elements in the first homology group $H_{1}(M)$, one sees that $H_{1}(M)=\mathbb{Z}^{2 g}$ (we refer the reader to [Jo] for details), which is a special case of the Hurewicz Theorem:

$$
H_{1}(M)=\frac{\pi_{1}\left(M, x_{0}\right)}{\left\{\left\langle\gamma \delta \gamma^{-1} \delta^{-1}\right\rangle: \gamma, \delta \in \pi_{1}\left(M, x_{0}\right)\right\}},
$$

where $\pi_{1}\left(M, x_{0}\right)$ denotes the first homotopy group. Thus, homology groups are always abelian, whilst homotopy groups are not if $g>1$.

Any simple closed curve which is homologically trivial, if it is not homotopically trivial, separates a torus from the surface, and we will say that it is on a collar of the surface.

The relation between differential forms and homology groups is given by Stokes' Theorem, which asserts that

$$
\int_{\partial C} \omega=\int_{C} d_{t} \omega
$$

if $\omega$ is a $j$-form, and $C$ is a $(j+1)$-chain, $j=0,1$.

In particular, it implies that $\int_{\gamma} b$ does not depend on the representative loop of $[\gamma] \in H_{1}(M)$. Also, it turns out that, when $b$ is exact, $\int_{\gamma} b=0$. The converse is true, since a primitive can be defined by integration.

By the Poincaré Lemma, a closed form on a simply connected Euclidean open set is exact, and then the integral of closed forms along a closed curve in a sufficiently small open set of $M$ is zero.

However, if one evaluates the integral of $b$ over a basis of $H_{1}(M)$, the results are not necessarily zero, and they are called periods of $b$. The number of incommensurable periods does not depend on the choice of the generators of $H_{1}(M)$ and is denoted by $\operatorname{rank}(b)$.

We can define the linear map $\operatorname{Per}(b): H_{1}(M) \rightarrow \mathbb{R}$ by

$$
[\gamma] \mapsto \int_{\gamma} b
$$

We will have that the image of $\operatorname{Per}(b)$ is a subgroup of $\mathbb{R}$ (this image is sometimes also denoted by $\operatorname{Per}(b))$. The rank of this group is $\operatorname{rank}(b)$; it is zero if and only if $b$ is exact.

We shall fix the periods $\int_{\gamma_{k}} b \doteq c_{k}, \int_{\delta_{k}} b \doteq d_{k}$, with $\gamma_{k}, \delta_{k}$ being the canonical generators of $H_{1}(M), k=1, \ldots, g$. 


\section{$1.2 \quad$ Minimal covering spaces}

We are given a surface $M$ of genus $g$ where a real smooth closed and non-exact 1 -form $b$ is defined.

There exists a compact polygon $P$ of $4 g$ sides pairwise identified in a way to yield $M$. All the vertices are identified to $x_{0} \in M$, and the sides are identified to the $2 g$ canonical generators of $\pi_{1}\left(M, x_{0}\right)$. Such a polygon $P$ will be called a fundamental domain.

Moreover, there exist $2 g$ translations (hyperbolic translations if $g>1$, and Euclidean translations if $g=1$ ) generating a group that, by acting on a fundamental domain, originates distinct and non-overlapping polygons for distinct elements, exhausting the universal covering space, and defining a projection $\Pi: D \rightarrow M$ (or $\left.\Pi: \mathbb{R}^{2} \rightarrow M\right)$. We denote these translations by $\gamma_{1}, \delta_{1}, \ldots, \gamma_{g}, \delta_{g}$. In fact, the path from $t_{0} \in D$ to $\gamma_{k}\left(t_{0}\right)$ is chosen to be a side of $P$; thus, it can be projected onto a generator of $\pi_{1}\left(M, x_{0}\right)$ (see [Fe]).

This group of translations is

$$
\operatorname{span}\left\{\gamma_{1}, \delta_{1}, \ldots, \gamma_{g}, \delta_{g}:\left[\gamma_{1}, \delta_{1}\right] \ldots\left[\gamma_{g}, \delta_{g}\right]=1\right\}
$$

where $[\gamma, \delta]$ represents the commutator $\gamma \delta \gamma^{-1} \delta^{-1}$.

We know that we can construct a primitive $B$ of $b$ by integration from a point on the universal covering space of $M$, the plane if $g$ is 1 , or the Poincaré disk $D$ if $g>1$. It turns out that we have $B\left(\gamma_{k}(t)\right)=B(t)+c_{k}$ and $B\left(\delta_{k}(t)\right)=B(t)+d_{k}$, for $k=1, \ldots, g$. In order to see this, if $t_{0}$ belongs to $D$ (or to the plane) and $\Pi\left(t_{0}\right)=x_{0}$, then for each $k$ we have

$$
B\left(\gamma_{k}(t)\right)=\int_{t_{0}}^{\gamma_{k}(t)} \Pi^{*}(b)=\int_{t_{0}}^{\gamma_{k}\left(t_{0}\right)} \Pi^{*}(b)+\int_{\gamma_{k}\left(t_{0}\right)}^{\gamma_{k}(t)} \Pi^{*}(b)=\int_{t_{0}}^{\gamma_{k}\left(t_{0}\right)} \Pi^{*}(b)+B(t)
$$

since $\Pi=\Pi \circ \gamma_{k}$ and, thus, $\Pi^{*}(b)=\left(\Pi \circ \gamma_{k}\right)^{*}(b)=\gamma_{k}^{*}\left(\Pi^{*}(b)\right)$; here $\Pi^{*}(b)$ denotes the pullback of $b$.

The same computation holds for $\delta_{k}$.

The numbers $\int_{t_{0}}^{\gamma_{k}\left(t_{0}\right)} \Pi^{*}(b)$ and $\int_{t_{0}}^{\delta_{k}\left(t_{0}\right)} \Pi^{*}(b)$ do not depend on $t_{0}$. Indeed, if $t_{0}^{\prime} \in D$

$$
\int_{t_{0}^{\prime}}^{\gamma_{k}\left(t_{0}^{\prime}\right)} \Pi^{*}(b)=\int_{t_{0}^{\prime}}^{t_{0}} \Pi^{*}(b)+\int_{t_{0}}^{\gamma_{k}\left(t_{0}\right)} \Pi^{*}(b)+\int_{\gamma_{k}\left(t_{0}\right)}^{\gamma_{k}\left(t_{0}^{\prime}\right)} \Pi^{*}(b)=
$$




$$
=-\int_{t_{0}}^{t_{0}^{\prime}} \Pi^{*}(b)+\int_{t_{0}}^{\gamma_{k}\left(t_{0}\right)} \Pi^{*}(b)+\int_{t_{0}}^{t_{0}^{\prime}} \gamma_{k}^{*}\left(\Pi^{*}(b)\right)=\int_{t_{0}}^{\gamma_{k}\left(t_{0}\right)} \Pi^{*}(b) .
$$

As is well-known, $\pi_{1}\left(M, x_{0}\right)$ is isomorphic to this group of translations. By Proposition 1.40 from [Ha], it is the group of deck transformations of the universal covering space. By means of this isomorphism, a translation $\gamma$ corresponds to a loop in $M$ which is the projection of a path connecting $t_{0}$ to $\gamma\left(t_{0}\right)$.

Definition 1.1. Let $\widetilde{M}$ be a covering space of $M$ and $\mathrm{D}$ be the group of deck transformations of $\widetilde{M}$. A function $B: \widetilde{M} \rightarrow \mathbb{R}$ is called a pseudoperiodic function if

$$
\text { for each } \sigma \in \mathrm{D} \text { such that } B(\sigma(t))=B(t), \forall t \in \widetilde{M} \text {, one has } \sigma=1 \text {. }
$$

With this definition, the function $B$ constructed on $D$ by integration is never a pseudoperiodic function. In fact, since $\pi_{1}\left(M, x_{0}\right)$ is not abelian, a non-trivial element $\sigma \in \pi_{1}\left(M, x_{0}\right)$ in the subgroup of the commutators satisfies $B(\sigma(t))=B(t)$, for all $t \in D$, regardless of the periods of $b$. However, if the genus is 1 and $\operatorname{rank}(b)$ is $2, B$ indeed defines a pseudoperiodic function in the plane.

Underneath (1.3), lies the fact that the integral of $\Pi^{*}(b)$ along a closed curve is zero. Therefore, we shall deal with another covering space where $B$ can still be defined by integration. This covering is obtained by considering the subgroup $G$ of $\pi_{1}\left(M, x_{0}\right)$ containing every element that annihilates the integral, that is, the kernel of the homomorphism $T: \pi_{1}\left(M, x_{0}\right) \rightarrow \mathbb{R}$ given by

$$
\gamma \mapsto \int_{\gamma} b
$$

By Theorem 1.38 of [Ha], we conclude the existence of a covering space $\left(\widetilde{M}, \widetilde{x_{0}}\right) \rightarrow$ $\left(M, x_{0}\right)$ such that $\pi_{1}\left(\widetilde{M}, \widetilde{x_{0}}\right)$ is isomorphic to $G$. This covering is unique up to homeomorphisms, and the basepoints can be omitted as $G$ is normal.

We call the covering $\widetilde{M}$ the minimal covering of $M$ (with respect to $b$ ), minimal in the sense that, if we want to construct a covering space on which a primitive of $b$ is defined, then $G$ must contain a copy of the fundamental group of such a covering space.

Of course this construction encompasses even the cases when $\operatorname{rank}(b)$ is not maximal.

By Proposition 1.39 of $[\mathrm{Ha}]$, for each pair of lifts of $x_{0}$ in $\widetilde{M}$, there is a deck transformation sending one to the other. Thereat, $\widetilde{M}$ is called a normal covering space. Moreover, the group D of deck transformations of $\widetilde{M}$ will be $\frac{\pi_{1}(M)}{G}$, which is finitely 
generated. Let us denote a minimal set of generators by $\left\{\sigma_{j}\right\}, j=1, \ldots, l$ (they are equivalence classes in $\left.\pi_{1}(M)\right)$.

We define $B$ by integration from a fixed $t_{0} \in \widetilde{M}$. Likewise (1.3), if $\sigma \in \mathrm{D}$, then it turns out that, for every $t \in \widetilde{M}$, we have

$$
B(\sigma(t))-B(t)=\int_{t_{0}}^{\sigma\left(t_{0}\right)} \Pi^{*}(b),
$$

which is equal to zero if and only if $\sigma$ is 1 .

Now, write

$$
B\left(\sigma_{j}(t)\right)=B(t)+b_{j}
$$

and notice that $b_{j}$ are rationally independent for $j=1, \ldots, l$, otherwise we would have a zero linear combination of the generators with integer coefficients.

The covering $\widetilde{M}$ is sometimes called a periodic manifold. By this we mean that D acts on $\widetilde{M}$ in such a way that: every $t \in \widetilde{M}$ has a neighborhood $U$ such that the only $\sigma \in \mathrm{D}$ with $\sigma(U) \cap U \neq \varnothing$ is the identity $\sigma=1$.

As desired, we have obtained a periodic manifold $\widetilde{M}$ and a pseudoperiodic primitive $B$. We denote the projections by $\Pi_{1}: D \rightarrow \widetilde{M}\left(\right.$ or $\Pi_{1}: \mathbb{R}^{2} \rightarrow \widetilde{M}$ ) and $\Pi_{2}: \widetilde{M} \rightarrow M$.

We will call $\Pi_{1}(P)$ a fundamental domain of $\widetilde{M}$, in the sense that $\cup_{\sigma \in \mathrm{D}} \sigma\left(\Pi_{1}(P)\right)=$ $\widetilde{M}$ and $\operatorname{int}\left(\Pi_{1}(P)\right) \cap \sigma\left(\Pi_{1}(P)\right)=\varnothing$ if $\sigma \neq 1$.

In the next section we give some examples of minimal covering spaces.

\subsection{Examples}

( $i$ ) Consider a surface of genus $g$ and a 1-form $b$ such that, for a fixed $k \in$ $\{1, \ldots, g\}$, the periods $c_{k^{\prime}}$ and $d_{k^{\prime}}$ are zero if $k^{\prime} \neq k$. Assume also that $\operatorname{rank}(b)$ is 1 , which means that there exist $p, q \in \mathbb{Z}$ such that $p c_{k}+q d_{k}=0$.

A covering surface $\widetilde{M}$, where a pseudoperiodic function $B$ is defined, is constructed as follows: consider the torus $T_{k}$ of the connected sum and the line $p x+q y=0$ in a plane that covers $T_{k}$. We cut along the projection $\beta$ on $M$ of this line, with small changes in order to avoid the disks that form the sum if necessary.

The result is a surface of genus $g-1$ with two boundary components, our fundamental domain where a primitive is defined by integration. Now, we glue infinitely many copies of this new surface vertically by identifying the boundaries as in Figure 6.1 (page 55) and extend $B$ by a translation $\tau$. Hence, the group $D$ is isomorphic to $\mathbb{Z}$.

Notice there is a path from $t$ to $\tau(t)$ projected onto another generator of the homology of $T_{k}$, a closed curve which has an odd intersection number with $\beta$ and, 
hence, a non-zero period.

We have a pseudoperiodic function $B$ and the covering is minimal. Indeed, the lift of a closed curve in $G$ must be in $\pi_{1}(\widetilde{M})$; otherwise, since Proposition 1.40 of [Ha] says that $\widetilde{M}$ is a normal covering, we would have a non trivial element $\sigma$ in the group of deck transformations of $\widetilde{M}$ such that $B(\sigma(t))=B(t)$, for every $t \in \widetilde{M}$.

This example extends the case when the cylinder is used in order to cover the torus in [BKNZ].

(ii) Consider a surface of genus $g$ and a 1-form $b$ such that, for a fixed $k \in$ $\{1, \ldots, g\}$, the periods $c_{k^{\prime}}$ and $d_{k^{\prime}}$ are zero if $k^{\prime} \neq k$. Also, assume that $\operatorname{rank}(b)$ is 2 , which means that $c_{k}$ and $d_{k}$ are incommensurable.

We cut along the generators of the homology with nonvanishing periods and we glue infinitely many copies of this surface with boundary by identifying them properly, in the sense that a pseudoperiodic primitive $B$ of $b$ is defined, and the result is the lollipops (or lifesavers). In Figure 2.1, page 15, we illustrate it for genus 2.

This example extends the case when the plane is used in order to cover the torus in $[\mathrm{BK}]$.

(iii) Consider the bitorus and $b$ a 1 -form with periods $c_{1}, d_{1}, c_{2}, d_{2}$. Assume that $\operatorname{rank}(b)$ is 2 and, furthermore, that $c_{1}, d_{1}$ are commensurable and so are $c_{2}, d_{2}$.

We now cut $M$ along two disjoint simple closed curves with zero period, each one in a different torus of the connected sum, obtaining a surface with four boundary components.

We glue copies of this surface in order to get the plumbing (see Figure 1.1) and a pseudoperiodic function $B$.

Notice that here and in Example $(i i)$, $\mathrm{D}$ is isomorphic to $\mathbb{Z} \times \mathbb{Z}$; it is generated by two translations.

\subsection{Compatibility conditions}

Definition 1.2 (Compatibility conditions). We say that a 1 -form $f \in \bigwedge^{1} C^{\infty}\left(M \times \mathbb{S}^{1}\right)$ belongs to $\mathbb{E}$ if for each $\xi \in \mathbb{Z} \backslash\{0\}$ and each closed smooth curve $\gamma$ in $\widetilde{M}$,

$$
\int_{\gamma} e^{-\xi B(s)} \hat{f}(s, \xi)=0
$$

and if for each closed smooth curve $\gamma$ in $M$,

$$
\int_{\gamma} \hat{f}(s, 0)=0
$$

Now, we will say a few words about this definition. 


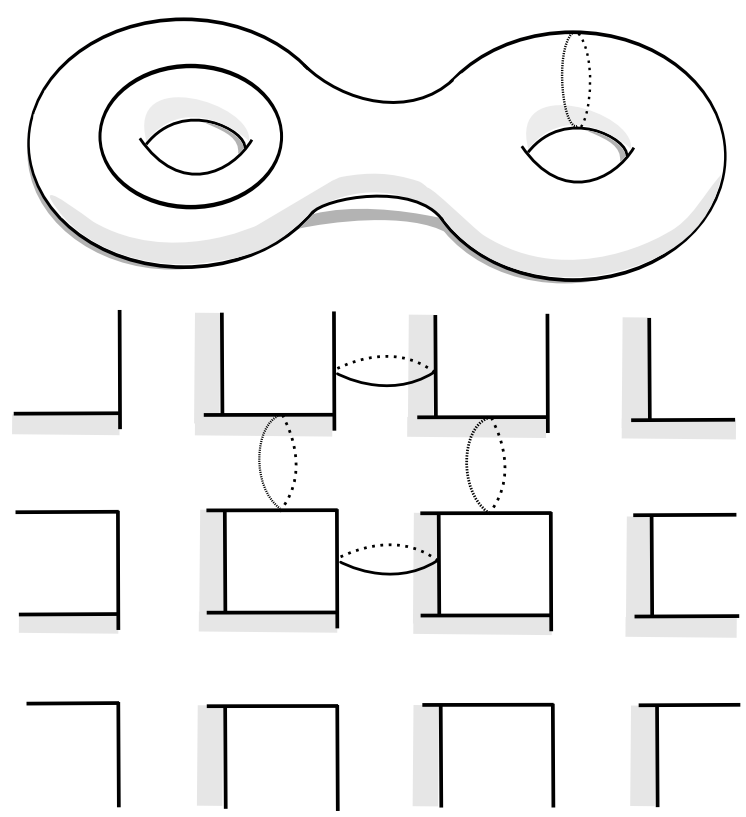

Figure 1.1: Cutting where the periods are zero

Under the conditions of Definition 1.2, we are using the pullback to $\widetilde{M}$ of the non-Fourier coefficients of $f$. For the sake of simplicity, we omit the pullbacks in the text from now on when their omission does not raise any doubts.

Also, note that this definition is independent of the choice of a primitive $B$.

Essentially, it says that the function $t \mapsto \int_{t_{0}}^{t} e^{-\xi B(s)} \hat{f}(s, \xi)$ for $t, t_{0} \in \widetilde{M}, t_{0}$ being fixed, is well-defined for each $\xi \in \mathbb{Z}$. Sometimes we write this as follows:

$$
e^{-\xi B(t)} \hat{f}(t, \xi) \text { is an exact } 1 \text {-form on } \widetilde{M} \text {, for every } \xi \in \mathbb{Z} \text {. }
$$

The conditions come from a computation concerning a necessary condition for a 1 -form to be in the image of the operator $\mathbb{L}$. Indeed, if we view (1.2) on $\widetilde{M}$, we can apply an integrating factor, and then

$$
d_{t}\left(e^{-\xi B(t)} \hat{u}(t, \xi)\right)=e^{-\xi B(t)} \hat{f}(t, \xi)
$$

In the proof of Theorem 2.1 we will give some examples of 1-forms satisfying these conditions.

Definition 1.3. We say that the operator (1.1) is globally solvable if given any 1-form $f \in \mathbb{E}$ there exists $u \in \mathscr{D}^{\prime}\left(M \times \mathbb{S}^{1}\right)$ such that $\mathbb{L} u=f$.

For our purposes, the next proposition will be fundamental.

Proposition 1.4. If $f \in \mathbb{E}$, then for each $\xi \in \mathbb{Z}, \xi \neq 0$, and each $t \in \widetilde{M}$, the numbers 


$$
\rho(\xi) \int_{t}^{\sigma(t)} e^{-\xi[B(s)-B(t)]} \hat{f}(s, \xi),
$$

with $\rho(\xi)=\left(e^{-\xi(B(\sigma(t))-B(t))}-1\right)^{-1}$, are independent of the translation $\sigma \in \mathrm{D}$ if $B(\sigma(t))-$ $B(t) \neq 0$.

Proof. Recall that, by (1.4), $B(\sigma(t))-B(t)$ depends only on $\sigma \in \mathrm{D}$.

Since $f \in \mathbb{E}$, the integral is independent of the path. Consider two translations, $\sigma_{1}$ and $\sigma_{2}$, with $B\left(\sigma_{1}(t)\right)-B(t)=b_{1} \neq 0$ and $B\left(\sigma_{2}(t)\right)-B(t)=b_{2} \neq 0$, and set $v(s)=e^{-\xi B(s)} \hat{f}(s, \xi)$. We have

$$
\int_{t}^{\sigma_{1} \sigma_{2}(t)} v=\int_{t}^{\sigma_{1}(t)} v+\int_{\sigma_{1}(t)}^{\sigma_{1} \sigma_{2}(t)} v
$$

and if we make the change of variables $s^{\prime}=\sigma_{1}^{-1}(s)$ in the last integral, we obtain

$$
\int_{\sigma_{1}(t)}^{\sigma_{1} \sigma_{2}(t)} v=e^{-\xi b_{1}} \int_{t}^{\sigma_{2}(t)} v .
$$

Hence,

$$
\int_{t}^{\sigma_{1} \sigma_{2}(t)} v=\int_{t}^{\sigma_{1}(t)} v+e^{-\xi b_{1}} \int_{t}^{\sigma_{2}(t)} v
$$

Similarly,

$$
\int_{t}^{\sigma_{2} \sigma_{1}(t)} v=\int_{t}^{\sigma_{2}(t)} v+e^{-\xi b_{2}} \int_{t}^{\sigma_{1}(t)} v
$$

As the group $D$ is abelian, we have the desired result.

Next, note that if we want to solve the system $\mathbb{L} u=f$, then by involutivity we must have $\mathbb{L}^{1} f=0$ if $f \in \mathbb{E}$; this also follows from Definition 1.2.

We point out that in [BK, BKNZ] the compatibility conditions for the second member $f \in \bigwedge^{1} C^{\infty}\left(M \times \mathbb{S}^{1}\right)$ are given by $\mathbb{L}^{1} f=0$ and the condition of Definition 1.2 when $\xi$ is equal to 0 . 


\section{CHAPTER 2}

A NECESSARY CONDITION FOR SOLVABILITY

We now state the result which allows us to conclude that certain systems are not globally solvable.

Theorem 2.1. Let $\widetilde{M}$ be a covering space of $M$ on which a pseudoperiodic primitive, $B$, of $b$ is defined. Suppose further that there exists a nonempty connected component, $\Omega$, of a sublevel or a superlevel such that $B$ is bounded on $\Omega$. Then the system $\mathbb{L} u=f$ is not globally solvable.

Sometimes we will restrict ourselves to the case of superlevels in order to keep the text clean, but all the claims are true also for sublevels.

The connectedness of the superlevels and sublevels in the minimal covering space is the necessary and the sufficient condition for the global solvability in $[\mathrm{CH}]$ for any given surface if $b$ is exact (the minimal covering being the surface itself), and in [BK, BKNZ] if $M$ is the torus and $b$ is closed, but not exact.

In the latter situation, there is a unique unbounded component of a superlevel, hence the non-connectedness of a superlevel implies the existence of a bounded component of superlevel, therefore, a superlevel having a component where $B$ is bounded.

What is remarkable now is that, in Chapter 3, we will have examples of global solvability on the bitorus in the presence of a disconnected superlevel in the minimal covering space (see also Example 2.4).

Our work in the Introduction was to find the periodic manifold where we can make the calculations in the proof of Theorem 2.1. It turned out to make a quotient of the universal covering space, although it can be convenient to have a condition there also.

For this, recall that we denote by $\Pi_{1}: D \rightarrow \widetilde{M}$ and $\Pi_{2}: \widetilde{M} \rightarrow M$ the projections. We call, for a moment, $B_{1}: D \rightarrow \mathbb{R}$ and $B_{2}: \widetilde{M} \rightarrow \mathbb{R}$ primitives of $b, B_{1}(t)=\int_{t_{0}}^{t} \Pi^{*} b$ and $B_{2}(t)=\int_{\Pi_{1}\left(t_{0}\right)}^{t}\left(\Pi_{2}\right)^{*} b$; and $\Omega_{r}^{1}, \Omega_{r}^{2}$ their respective $r$-superlevels (analogously for 
the plane if $g$ is 1 ).

We claim the following

\section{Proposition 2.2.}

(i) There is a component of $\Omega_{r}^{1}$ on which $B_{1}$ is unbounded;

(ii) $B_{1}$ is bounded on some component of $\Omega_{r}^{1}$ if and only if $B_{2}$ is bounded on some component of $\Omega_{r}^{2}$;

(iii) $\Omega_{r}^{1}$ is connected if and only if $\Omega_{r}^{2}$ is connected.

Proof. ( $i$ ) Consider a fundamental polygon $P$ and $\sigma$ a generator of the group of deck transformations, with $s=\int_{t}^{\sigma(t)} \Pi^{*} b$ different from zero, for $t \in D$ (we may assume that $s$ is positive). Since $M$ is compact, $B_{1}(t) \geqslant K$, for some $K \in \mathbb{R}$ and for all $t \in P$. Now, there is a smallest integer $n_{0}$ such that $B_{1}\left(\sigma^{n_{0}}(t)\right) \geqslant K+n_{0} . s>r$, for all $t \in P$. The set $\cup_{n \geqslant n_{0}} \sigma^{n}(P) \subseteq \Omega_{r}^{1}$ is connected and $B_{1}$ is unbounded on it, hence it suffices to take the connected component containing this set.

(ii) Assume $B_{1}$ is bounded on a component $O_{1}$ of $\Omega_{r}^{1}$.

Any path in the component of $\Omega_{r}^{2}$ containing $\Pi_{1}\left(O_{1}\right)$ and starting in $\Pi_{1}\left(O_{1}\right)$ can be lifted to $O_{1}$ since, for $s=\Pi_{1}\left(s_{1}\right)$ in this path, we have $B_{2}(s)=\int_{\Pi_{1}\left(t_{0}\right)}^{s}\left(\Pi_{2}\right)^{*} b=$ $\int_{t_{0}}^{s_{1}} \Pi^{*} b=B_{1}\left(s_{1}\right)$. Hence, $\Pi_{1}\left(O_{1}\right)$ is a component of $\Omega_{r}^{2}$ on which $B_{2}$ is bounded.

Similarly, the components of the preimage by $\Pi_{1}$ of a component of $\Omega_{r}^{2}$ on which $B_{2}$ is bounded are components of $\Omega_{r}^{1}$, and $B_{1}$ is bounded on them.

(iii) If $\Omega_{r}^{2}$ is disconnected, then it is immediate that so is $\Omega_{r}^{1}$. Now, suppose $\Omega_{r}^{2}$ is connected. Then, $\Omega_{r}^{2}$ is the image by $\Pi_{1}$ of the component $O$ obtained in $(i)$.

In fact, if we repeat the same proof of $(i)$ in $\widetilde{M}$, this time by using the fundamental domain $\Pi_{1}(P)$ and a non-trivial $\sigma \in \mathrm{D}$, we see that $\Pi_{1}(O)$ covers an entire fundamental domain and, consequently, the generators of the homotopy of $\widetilde{M}$ with a basepoint.

If $\Omega_{r}^{1}$ has another component, called $O^{\prime}$, it must be projected in $\Omega_{r}^{2}$ too, and then we would have a point in $O^{\prime}$ and a point in $O$ with the same projection in $\Omega_{r}^{2}$. Hence, $O^{\prime}$ contains a translate of $O$. This yields a contradiction, because there are points in $\Pi_{1}(O)$ whose preimages can always be connected in $O$.

Remark 2.3. The component $O$ from $(i)$ is unbounded in D. Its image by the projection $\Pi_{1}$ is, by (ii), a component of superlevel on which $B_{2}$ is unbounded.

By following (iii), we infer that $\Pi_{1}(O)$ contains infinitely many translates of a fundamental domain, and hence we have a right to refer to this component as unbounded. However, we will not specify here a metric for $\widetilde{M}$; recall that, differently from the discussed results, we have set our hypothesis for the non-solvability as the boundedness of $B_{2}$ on a component. 
Example 2.4. In Chapter 3, a bitorus and a 1-form b will be constructed from two tori $T_{1}$ and $T_{2}$ where the 1 -forms $\omega_{1}=c_{1} d \theta+d_{1} d \phi$ and $\omega_{2}=c_{2} d \theta+d_{2} d \phi$ are respectively defined. We can choose the periods to be incommensurable, that is, so as to have $\operatorname{rank}(b)$ equals 4 . Consider $f(x, y, z)=-z^{2}$, where $(x, y, z)$ are coordinates on $\mathbb{R}^{3}$.

A 1-form on the bitorus is defined by choosing sufficiently small open sets $N_{i}$ in $T_{i}$ such that on each one of them the 1-forms previously defined coincide with $d f$ and such that in the collar there is an $\mathbb{S}^{1}$ where $f$ is zero (an illustration is in Figure 3.2).

Consider a strip $S$ of level sets close enough this $\mathbb{S}^{1}$. We lift such level sets to segments in $D$ the union of which can be translated by a non-trivial element of the commutator of $\pi_{1}(M)$ so that the resulting set is unbounded and covers $S$. Thus, we have an unbounded component of a superlevel on which $B$ is bounded.

Notice that there are sublevels with more than one unbounded component. Indeed, just cut along the zero set of $f$ in the collar and observe the planes, with boundary components, covering the tori $M_{i}$ with boundary, $i=1,2$, and on which we can respectively define a primitive of $\left.b\right|_{M_{i}}, i=1,2$. A boundary component of such a plane is part of a level set of the primitive there defined. In both planes we detect unbounded components of sublevels whose preimage in D will be disjoint. By Proposition 2.2(iii), these sublevels are disconnected in $\widetilde{M}$ too.

In the next section we emphasize two important results.

\subsection{Auxiliary lemmas}

Essentially, Lemma 2.5 below is in $[\mathrm{Ar}]$. We point out that, in there, a different covering is considered, namely a covering space of $M$ embedded in $\mathbb{R}^{N}$, for a sufficiently large $N$, in certain cases, in particular when $b$ is Morse.

Lemma 2.5. Under the hypotheses of Theorem 2.1, the translates of the component $\Omega$ by non-trivial elements of $\mathrm{D}$ are pairwise disjoint.

Therefore, such a component can be projected diffeomorphically on the surface, and this will be the key step to the proof of Theorem 2.1.

Proof. Take $\sigma \in \mathrm{D}$ such that $\sigma \neq 1$. It is enough to show that $\sigma(\Omega)$ is disjoint from $\Omega$.

If $\Omega$ is a component of the $r$-superlevel, then $\sigma(\Omega)$ is a connected set inside the $(r+s)$-superlevel, with $s=\int_{t}^{\sigma(t)}\left(\Pi_{2}\right)^{*} b$, for any $t \in \widetilde{M}$. Since $s \neq 0$, we may suppose $s>0$. Therefore, $\sigma(\Omega)$ is inside a component of the $r$-superlevel.

If $\sigma(\Omega)$ is not disjoint from $\Omega$, then we must have $\sigma(\Omega) \subset \Omega$. By iterating this, we deduce that $\sigma^{n}(\Omega) \subset \Omega$, for $n \in \mathbb{N}$.

Since $\sigma^{n}(\Omega)$ is contained in the $(r+s . n)$-superlevel, we have a contradiction with the fact that $B$ is bounded on $\Omega$. 
We also make use of the following a priori estimates, a variant of a classical lemma of Hörmander's and based on [Tr2].

Lemma 2.6. If $\mathbb{L}$ is globally solvable, there exist constants $C>0$ and $m \in \mathbb{N}$ so that

$$
\left|\int_{\widetilde{M} \times \mathbb{S}^{1}} f \wedge g \wedge d x\right| \leqslant C\|f\|_{m}\left\|\mathbb{L}^{1} g\right\|_{m}
$$

for all $f \in \mathbb{E}$ (see Definition 1.2) and $g \in \bigwedge^{1} C_{c}^{\infty}\left(\widetilde{M} \times \mathbb{S}^{1}\right)$.

Proof. Let $u$ be a distribution on $M \times \mathbb{S}^{1}$ such that $\mathbb{L} u=f$. Then

$$
\int f \wedge g \wedge d x=-\int u \wedge \mathbb{L}^{1} g \wedge d x
$$

Consider in $\bigwedge^{1} C_{c}^{\infty}\left(\widetilde{M} \times \mathbb{S}^{1}\right) \doteq \Theta$ the topology defined by the semi-norms $v \mapsto$ $\left\|\mathbb{L}^{1} v\right\|_{m}$ and set $\Theta^{*} \doteq \frac{\Theta}{\operatorname{ker} \mathbb{L}^{1}}, m \in \mathbb{N}$.

When we form this quotient, the family of semi-norms becomes separating. Therefore, $\Theta^{*}$ is a metrizable topological vector space.

Since $\mathbb{E}$ is a closed linear subspace inside a Fréchet space, $\mathbb{E}$ is also a Fréchet space.

Now, (2.1) gives us a bilinear form on $\mathbb{E} \times \Theta^{*}$ that is separately continuous. By ([Tr1], p.354), we have a jointly continuous bilinear functional and, thus, the result.

We shall next construct sequences of 1-forms $f_{n} \in \mathbb{E}$ and $g_{n} \in \bigwedge^{1} C_{c}^{\infty}\left(\widetilde{M} \times \mathbb{S}^{1}\right)$ that violate the inequality above when $n$ goes to infinity.

\subsection{Construction of the forms and proof}

We proceed with a construction inspired by the notes of the lecture Solvability of a Model in the Theory of Complexes of Pseudodifferential Operators, given by François Treves at the Universidade Federal de Pernambuco, Brazil, and written in 1977 by Jorge Hounie.

We call $\Omega$ a component of a superlevel in $\widetilde{M}$ where $B$ is bounded, say the $r_{2^{-}}$ superlevel. Consider levels $r$ and $r_{1}$ both smaller than the supremum of $B$ on $\Omega$, with $r>r_{1}>r_{2}$.

Set $\Gamma^{s}=\{p \in \Omega: B(p)>s\}$. We have $\Gamma^{r} \subset \Gamma^{r_{1}} \subset \Omega$, and all of these sets are disjoint from their translates.

By Urysohn's Lemma, define a smooth function $F$ with compact support in $\overline{\Gamma^{r_{1}}}$ and identically 1 in $\overline{\Gamma^{r}}$. Put $f_{0} \doteq d_{t} F$. We deduce that

$$
B\left(\operatorname{supp}\left(f_{0}\right)\right) \leqslant r
$$


The covering $\widetilde{M}$ is path-connected, hence there exists a smooth path $\gamma$ joining a point $P_{1}$ in a component of the $r$-superlevel on which $B$ is unbounded (see Remark 2.3) to a point $P_{2} \in \Gamma^{r}$.

Consider small balls centered at $P_{1}$ and $P_{2}$ such that $B(t)>r+\varepsilon$ on them, for some $\varepsilon>0$. We can choose $P_{1}$ so that the extension of $F$ by translations vanishes in the ball centered at it, since this unbounded component covers a fundamental domain.

Let $t_{1}$ and $t_{2}$ be local coordinates around $\gamma$, with $t_{1}$ being tangent to $\gamma$.

Define $W=\partial_{1}$ a unit tangent vector field in a neighborhood of $\gamma$, and let $\theta$ be a function defined on this neighborhood such that $\partial_{1} \theta=1$. We construct the 1 -form $v_{0}$ by

$$
v_{0}(t)=g(\theta(t)) h(t) d t_{2}
$$

where $h$ is a function which is strictly positive in a neighborhood of $\gamma$, vanishes outside of another neighborhood $U$ of $\gamma$, and satisfies $W h=\partial_{1} h=0$.

Take now $g \in C_{c}^{\infty}(\mathbb{R})$ satisfying $g(s)=1$, if $\theta\left(P_{1}\right)<s<\theta\left(P_{2}\right)$. Moreover, assume that $g$ has support in $\left[\theta\left(P_{1}\right)-\varepsilon^{\prime}, \theta\left(P_{2}\right)+\varepsilon^{\prime}\right]$, with $\varepsilon^{\prime}$ and $U$ chosen so as to make the support of $t \mapsto h(t) g^{\prime}(\theta(t))$ lie inside those balls where $B$ is greater than $r+\varepsilon$. We illustrate these constructions in Figures 2.1 and 2.2.

A computation gives

$$
d_{t} v_{0}(t)=h(t) g^{\prime}(\theta(t)) d t_{1} \wedge d t_{2}
$$

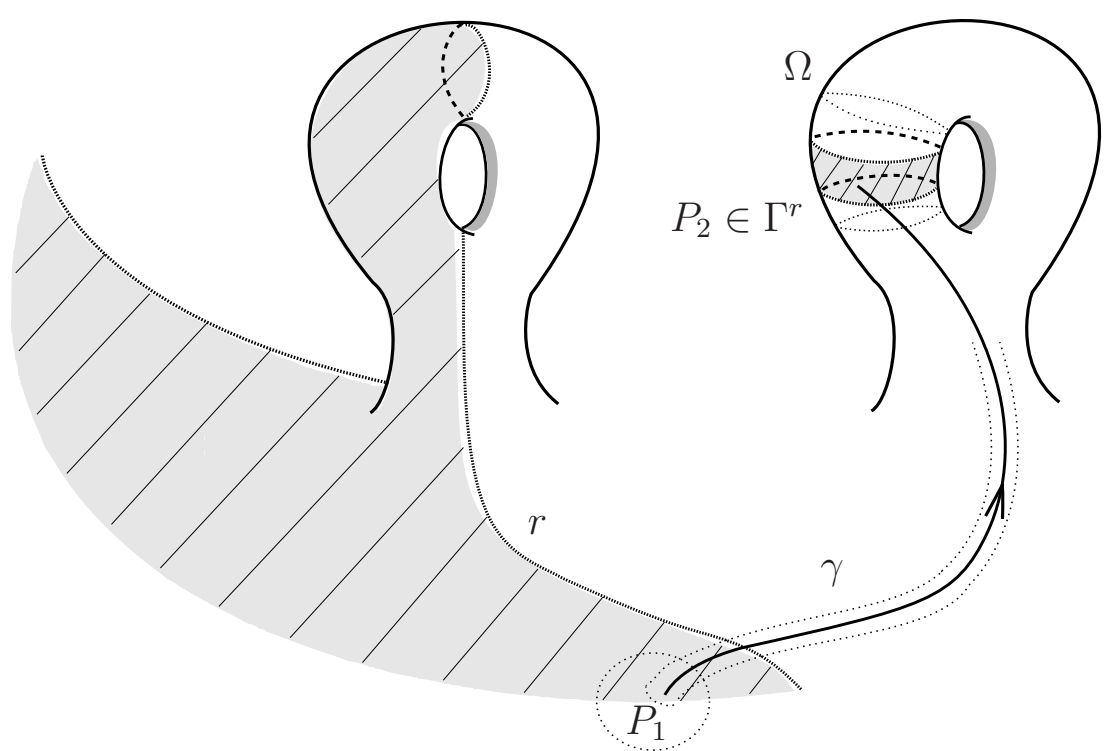

Figure 2.1: Construction of $\gamma$ 


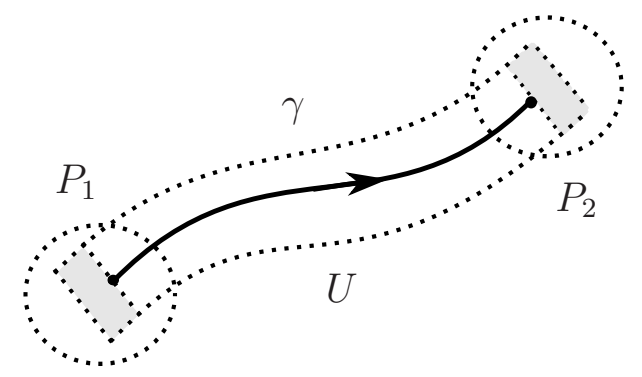

Figure 2.2: Support of $d_{t} v_{0}$

Finally we define $f_{n}(t, x)=e^{i n x+n B(t)} f_{0}(t)$ and $g_{n}(t, x)=e^{-i n x-n B(t)} v_{0}(t)$ (both $f_{n}$ and $g_{n}$ with only one non-zero Fourier coefficient).

Notice that $f_{n}$ can be defined on the surface and extended to the covering $\widetilde{M}$ by translations. This is not just extending $f_{0}$, but is still true that $e^{-n B(t)} \widehat{f}_{n}(t, n)$ is exact on $\widetilde{M}$, for all $n \in \mathbb{N}$. In fact, if $t \in \widetilde{M}$ and $\sigma_{j} \in \mathrm{D}$,

$$
\widehat{f}_{n}\left(\sigma_{j}(t), n\right)=\widehat{f}_{n}(t, n)=e^{n\left(B\left(\sigma_{j}(t)\right)-b_{j}\right)} f_{0}(t)
$$

and then $e^{-n B(t)} \widehat{f}_{n}(t, n)=d_{t} \tilde{F}(t)$, for each $n$, where $\tilde{F}$ is a smooth function that is a multiple of $F$ on each translate of $\Omega$.

Hence, $f_{n} \in \mathbb{E}$, and we can apply the inequalities of Lemma 2.6.

We have

$$
\mathbb{L}^{1} g_{n}(t, x)=e^{-i n x-n B(t)} d_{t} v_{0}(t),
$$

and by (2.2),

$$
\left\|f_{n}\right\|_{m} \leqslant C_{m} n^{m} e^{n r}
$$

Therefore, $\left\|f_{n}\right\|_{m}\left\|\mathbb{L}^{1} g_{n}\right\|_{m} \leqslant C_{m}^{\prime} n^{2 m} e^{-n \varepsilon}$, which goes to 0 when $n$ goes to infinity. However, by Stokes' Theorem,

$$
\int f_{n} \wedge g_{n} \wedge d x=2 \pi \int d_{t} \tilde{F} \wedge v_{0}=2 \pi \int \tilde{F}(t) h(t) g^{\prime}(\theta(t)) d t_{1} \wedge d t_{2}
$$

and the last integral is strictly negative. In fact, we have constructed $t \rightarrow h(t) g^{\prime}(\theta(t))$ to be different from 0 inside two balls. In one of these balls this function is positive, but on it $F$ was assumed to be zero. In the other one there is an open set where this function is negative and $F$ is equal to 1.

This contradiction leads us to conclude that there is $f \in \mathbb{E}$ so that the equation $\mathbb{L} u=f$ does not have solution on $M \times \mathbb{S}^{1}$. 


\subsection{Another proof}

In this section we describe a different way to show that our system is not globally solvable in $M \times \mathbb{S}^{1}$, under the stronger assumption that, in $\widetilde{M}$, there is a bounded component $\Omega$ of an $r_{1}$-superlevel.

Recall that $M$ is a surface of genus $g$, and this component can be projected diffeomorphically on the surface.

Again consider a level $r$ smaller than the supremum of $B$ on $\Omega$, with $r>r_{1}$.

The set $\Gamma=\{p \in \Omega: B(p)>r\}$ is also projected diffeomorphically on $M$. In addition, we can suppose that $r$ is a regular level, hence its projection by $\Pi_{2}: \Omega \rightarrow M$ consists of a finite number of smooth closed curves embedded in the surface.

This new construction exhibits explicitly the 1-form $f \in \mathbb{E}$ for which there is not even a distribution $v$ solving the system. We proceed inductively, and this $f$ will be obtained from the one that is constructed for the torus, in [BK, BKNZ].

We have three possibilities for these closed curves:

(i) There is a homotopically trivial curve $C$.

In this case, we cut $M$ along a smooth closed curve in a collar of the surface and avoiding $C$. The result is two surfaces of lower genus each one with one boundary component. Pick the surface which contains $C$. It may be the case that the region determined by $C$ does not contain a projection of a component of a superlevel, but then we proceed by working with a projection of a component of a regular sublevel inside this region. We keep calling $\Gamma$ this component.

(ii) There is a curve that represents a trivial element of $H_{1}(M)$, but not homotopically trivial curves.

In this case, we choose one of these curves and cut along a curve homotopic to it which is in $\Pi_{2}(\Omega)$, but not in $\Pi_{2}(\Gamma)$. The result is two surfaces of lower genus each one with one boundary component. Pick the one which contains $\Pi_{2}(\Gamma)$.

(iii) They do not represent trivial elements of $H_{1}(M)$.

Now, we choose one of these curves and cut along a curve homotopic to it which is in $\Pi_{2}(\Omega)$, but not in $\Pi_{2}(\Gamma)$. The result is a surface of genus $g-1$ with two boundary components.

We will construct a smooth closed 1-form $b_{0}$ on $M_{0}$, a surface of genus less than $g$, as follows.

In the obtained surface with boundary, consider $V \subset V_{1} \subset V_{2} \subset V_{3}$ small open neighborhoods of the boundary components.

We define a smooth function $\phi$ equals 0 on $\overline{V_{1}}$ and equals 1 on $\overline{V_{3} \backslash V_{2}}$. In any of the above cases, we can assume that a primitive of $b$ is defined on $V_{3} \backslash \bar{V}$ and that 
$V_{3}$ is disjoint from $\Pi_{2}(\Gamma)$. Then we set

$$
b_{0}= \begin{cases}b(t), & t \notin \overline{V_{2}} \\ d_{t}(\phi B)(t), & t \in V_{3} \backslash \bar{V} \\ 0, & t \in V_{1} .\end{cases}
$$

Hence, $b_{0}$ can be considered as a smooth closed 1-form defined on $M_{0}$.

In the minimal covering space $\widetilde{M}_{0}$ of $M_{0}$ with respect to $b_{0}$, a copy of $\Gamma$, which we call $\Gamma_{0}$, is a bounded component of a superlevel (or a sublevel) of a primitive $B_{0}$ of $b_{0}$.

By the inductive hypothesis, the system $\mathbb{L}_{0}$ in $M_{0} \times \mathbb{S}^{1}$ with $b_{0}$ does not have a solution for a certain $f_{0}$.

Notice that when we use the induction hypothesis for the first time, $\Gamma_{0}$ is a bounded subset of the plane or of the cylinder, according to the rank of $b_{0}$. By following the proofs of $[\mathrm{BK}]$ or $[\mathrm{BKNZ}]$, respectively, a smooth 1 -form $f_{0}$ is constructed in a way that the Fourier coefficients $\{\hat{v}(t, \xi)\}_{\xi \in \mathbb{Z}}$ of the candidate to a solution does not have tempered growth at a point $t^{*}$. This point can be chosen inside $\Pi\left(\Gamma_{0}\right)$ as the point of maximum or minimum of $B_{0}$ there.

Another important fact is that the Fourier coefficients $\{\hat{v}(t, \xi)\}_{\xi \in \mathbb{Z}}$ do have rapid decay on a neighborhood of $\partial\left(\Pi\left(\Gamma_{0}\right)\right)$ since, there, $B_{0}(t)$ is either strictly greater or strictly less than $B_{0}\left(t^{*}\right)$.

Now we are ready to define a smooth 1 -form $f$ on $M \times \mathbb{S}^{1}$. Let $\psi$ be a cut-off function equals 1 on $\Pi\left(\Gamma_{0}\right)$ and with support in a sufficiently small neighborhood of it in a way that $d_{t} \psi$ is different from zero only at the points where the coefficients $\{\hat{v}(t, \xi)\}_{\xi \in \mathbb{Z}}$ decay.

We assume that this neighborhood of $\Pi\left(\Gamma_{0}\right)$ is disjoint from $V_{3}$. Hence, $b_{0}(\cdot) \psi(\cdot)$ can be assumed as defined on $M$ and there $b_{0}(t) \psi(t)=b(t) \psi(t)$.

Therefore, on $M$, we have

$$
\begin{gathered}
d_{t} \hat{v}(t, \xi) \psi(t)+\hat{v}(t, \xi) d_{t} \psi(t)-\xi b(t) \wedge(\hat{v}(t, \xi) \psi(t))= \\
=\widehat{f}_{0}(t, \xi) \psi(t)+\hat{v}(t, \xi) d_{t} \psi(t) \doteq \hat{f}(t, \xi) .
\end{gathered}
$$

Notice that the coefficients $\{\hat{f}(t, \xi)\}_{\xi \in \mathbb{Z}}$ define an element of $\mathbb{E}$. Indeed, it is plain that $\left\{\widehat{f}_{0}(t, \xi) \psi(t)\right\}_{\xi \in \mathbb{Z}}$ have rapid decay and, according to our choice of $\psi$ and $v$, so do $\left\{\hat{v}(t, \xi) d_{t} \psi(t)\right\}_{\xi \in \mathbb{Z} \text {. }}$

Finally, $\{\hat{v}(t, \xi) \psi(t)\}_{\xi \in \mathbb{Z}}$ should be the Fourier coefficients of a solution to $\mathbb{L} u=$ $f$, but since $\psi$ is equal to 1 at $t^{*}$, they do not define a distribution on $M \times \mathbb{S}^{1}$. 


\section{CHAPTER 3}

\section{EXAMPLES OF GLOBALLY SOLVABLE SYSTEMS}

We consider here some particular constructions of 1-forms for our system that will motivate some general statements.

\subsection{Foliations}

A foliation $\mathscr{F}$ of codimension 1 on a surface $M$ will be a smooth atlas, maximal with respect to the following property:

If $(U, \phi)$ denotes a local chart such that $\phi(U)=U_{1} \times U_{2}$, where $U_{1}$ and $U_{2}$ are open intervals of $\mathbb{R}$, and if $(V, \psi)$ is another local chart, the change of coordinates $\psi \circ \phi^{-1}: \phi(U \cap V) \rightarrow \psi(U \cap V)$ is of the type $\psi \circ \phi^{-1}(x, y)=\left(h_{1}(x, y), h_{2}(y)\right)$; that is, it preserves horizontal lines.

The sets $\phi^{-1}\left(U_{1} \times\{c\}\right)$ in $\mathbb{R}^{2}$ are called the plaques of the foliation. Two points $m, m^{\prime} \in M$ are said to be in the same leaf if there is a chain of plaques $P_{1}, \ldots, P_{k}$, with $P_{i} \cap P_{i+1} \neq \varnothing$, for $i=1, \ldots, k-1, m \in P_{1}$, and $m^{\prime} \in P_{k}$. A leaf is a connected and immersed 1-dimensional manifold in $M$ (not embedded, in general).

We will study foliations given by Morse forms. It is important to remark that, in this section, many things can be done by requiring only that the smooth closed 1 -form $b$ has isolated singularities.

By a Morse 1-form $b$ we mean a smooth closed 1-form whose local primitives have only non-degenerate critical points (the primitives defined on a covering space have the same property). Then, there will be a local chart in a neighborhood of a critical point $p$ such that $B \circ \phi^{-1}(x, y)= \pm x^{2} \pm y^{2}$. The set of the singular points, $\operatorname{sing}(b)$, is finite.

Claim. The set of Morse 1-forms is an open and dense subset of the closed 1-forms. 
Proof. Consider for a moment a 1 -form $b$ an element in $C^{\infty}(M, T M)$ (equipped with the Whitney topology), where $T M$ represents the tangent bundle. We write $b$ locally as $b(t)=\left(t,\left(\partial_{1} B(t), \partial_{2} B(t)\right)\right)$.

Also consider domains of local charts $U_{j}, V_{j}$, with $U_{j} \subset V_{j}$ and $j \in J=$ $\{1, \ldots, n\}$, such that $\left\{U_{j}\right\}_{j \in J}$ covers $M$ and $\phi_{j}: M \rightarrow \mathbb{R}$ are defined satisfying $\phi_{j}\left(U_{j}\right)=1$ and $\phi_{j}\left(V_{j}^{c}\right)=0$, for $j \in J$.

Define $F: M \times \mathbb{R}^{2 n} \rightarrow \mathbb{R}$ by $F(t, A)=\sum_{j \in J}\left(\lambda_{j}^{(1)} t_{1}+\lambda_{j}^{(2)} t_{2}\right) \phi_{j}(t)$, where the parameters $\lambda_{j}^{(1)}, \lambda_{j}^{(2)} \in \mathbb{R}$ give the vector $A$.

Now set $\psi: M \times \mathbb{R}^{2 n} \rightarrow T M$ as $\psi(t, A)=b(t)+\left(d_{t} F\right)(t, A)$ and consider the inclusion $i: M \hookrightarrow T M$.

With this construction, we have that $D_{(t, A)} \psi$ is surjective, for $(t, A) \in M \times \mathbb{R}^{2 n}$.

Let $A$ be a regular value of the restriction of the projection $M \times \mathbb{R}^{2 n} \rightarrow \mathbb{R}^{2 n}$ to $\psi^{-1}(i(M))$ (Sard's Theorem asserts that such vectors are everywhere dense in $\mathbb{R}^{2 n}$ ). Then

$$
T_{q} \psi^{-1}(i(M))+T_{q}(M \times\{A\})=T_{q}\left(M \times \mathbb{R}^{2 n}\right),
$$

where $q$ is such that $\psi(q)=\psi(p, A) \in i(M)$, for some $p \in M$. If we apply $D_{q} \psi$ above, then it follows that $T_{\psi(q)} i(M)$ supplements $D_{q} \psi\left(T_{q}(M \times\{A\})\right)$.

Now, define a closed 1 -form $\psi_{A}$ on $M$ by $t \mapsto \psi(t, A)$, which can be taken close enough to $b$.

Finally, notice that $\psi_{A}$ is Morse. Indeed, call $\Psi$ one of its local primitives and let $p \in M$ be a critical point of $\psi_{A}$. If we consider $q=(p, A)$, since $D_{q} \psi\left(T_{q}(M \times\{A\})\right)=$ $D_{p} \psi_{A}\left(T_{p} M\right)=\left\{\left(v, \operatorname{Hess}_{p} \Psi(v)\right), v \in T_{p} M\right\}$ and $T_{(p, 0)} i(M)=\left\{(v,(0,0)), v \in T_{p} M\right\}$, we have that $p$ is a non-degenerate critical point.

A Morse 1-form $b$ defines a line field $P(q)=\left\{v \in T_{q}(M): b_{q}(v)=0\right\}$ if $q$ is not in $\operatorname{sing}(b)$ (see $[\mathrm{Ca}])$.

Since $P$ is a line field, it is involutive, and then, by Frobenius' Theorem, it gives rise to a foliation $\mathscr{F}$ in $M \backslash \operatorname{sing}(b)$ (the fact that $b$ is closed also guarantees the involutivity, since an equivalent condition for this is given by $d b \wedge b=0$ ).

It will be important here to have an orientation for the line field and, hence, for this foliation. We say that $\mathscr{F}$ is orientable if there exists a vector field $X$ on $M \backslash \operatorname{sing}(b)$ such that $P(q)$ is generated by $X(q)$. Locally, if $b=b_{1} d t_{1}+b_{2} d t_{2}$, we define the vector $X(q)=\left(-b_{2}(q), b_{1}(q)\right)$ (in the coordinates of the tangent space to $\left.q\right)$.

We may consider the orbits $x(s)$ of this vector field also in $D$ (or in $\widetilde{M}$ ), and it turns out that, in there, one has $x^{\prime}(s) \cdot \nabla B(x(s))=0$, which means that $B$ is constant on them, and hence a component of a level set of $B$ is sent onto a leaf by the canonical 
projection. Conversely, a leaf can be lifted to a component of a level set in the covering space.

Considering a small neighborhood of a saddle point $p$ (in $M$ or in a covering space), there are two leaves having the saddle as $\omega$-limit set (we say that they are arriving at $p$ ) and two having the saddle as $\alpha$-limit set (we say that they are issuing from $p$ ). These four local leaves are called separatrices of $p$.

The foliation $\mathscr{F}$ has interesting properties. There are three types of leaves: compact, compactifiable and non-compactifiable. Here the terminology and the main facts are based on [Far] and [Gel].

\section{Definition 3.1.}

A compact leaf $\gamma$ is a leaf diffeomorphic to $\mathbb{S}^{1}$. Each component of the union of the compact leaves is called a maximal component.

A leaf $\gamma$ is called compactifiable if it is not compact and $\gamma \cup \operatorname{sing}(b)$ is a compact subset of $M$. Otherwise, we call it a non-compactifiable leaf. Each component of the union of the non-compactifiable leaves is called a minimal component.

We next recall some substantial results of this theory.

Proposition 3.2. If $b$ is Morse and $\gamma$ is a compact leaf, then $\gamma$ has a neighborhood $U$ in $M$ consisting of compact leaves and diffeomorphic to the annulus $\gamma \times(-\varepsilon, \varepsilon)$. Moreover, $B$ is defined on $U$, has only regular points and each leaf corresponds to a different level set.

Proof. We start by taking a tubular neighborhood of $\gamma$ in $M$, which is diffeomorphic to a cylinder. Any homotopically non-trivial simple closed curve in this neighborhood is homotopic to $\gamma$, hence $B$ is defined there and is assumed to be zero on $\gamma$.

Locally, every point of $\gamma$ is in a sufficiently small open set without critical points, and then we can assume these sets to be foliated by pairwise different levels of $B$.

Using the compactness of $\gamma$, we construct a neighborhood $U^{\prime}$ of $\gamma$ as a finite union of these open sets. Therefore, there exists $\varepsilon>0$ such that $B^{-1}(s)$ has a component inside $U^{\prime}$, for $|s|<\varepsilon$, which is a compact manifold. Finally, take $U=B^{-1}(-\varepsilon, \varepsilon) \cap$ $U^{\prime}$.

Therefore, the maximal components are open sets of $M$. The minimal components are open sets too. Essentially, it is necessary to prove that the union of compact leaves and the compactifiable ones is a closed set in $M \backslash \operatorname{sing}(b)$, and this is consequence of Theorem 3.2 from [Hae]. Thus, we have

Proposition 3.3. The boundary of the maximal or the minimal components consists of compactifiable leaves and singular points. 
There are just finitely many numbers of compactifiable leaves. In fact, for any such leaf, a singularity is in its closure and, then, must be a saddle point. But a saddle point is in the closure of at most four compactifiable leaves.

The closure of a compactifiable leaf is a closed arc connecting two saddles or a closed curve containing a saddle.

Proposition 3.4. If $\mathscr{F}$ has a non-compactifiable leaf $L$, then $\operatorname{rank}(b) \geqslant 2$.

Proof. Let $L$ be a non-compactifiable leaf. There exists a regular point $q$ such that $q \in \bar{L} \backslash L$. Consider a small neighborhood $U$ of $q$ and a local system of coordinates $(x, y)$ such that on $U$ we write $b=d y$. Hence, $L \cap U$ contains pairwise disjoint segments $y=c_{n}$, with $c_{n} \in \mathbb{R}$ and $n \in \mathbb{N}$. Now, we construct a simple closed curve $\gamma_{m, n}$ in $M$ such that $\int_{\gamma_{m, n}} b=c_{m}-c_{n}$, and this number can be taken small enough. This is not possible if $\operatorname{rank}(b)$ is 1 .

We restrict ourselves to stating the final result of this section and addressing the reader to $[\operatorname{Im} 1]$.

Theorem 3.5. Each non-compactifiable leaf is dense in its minimal component.

\subsection{Construction of 1 -forms}

We will study first the global solvability for a class of examples. There is an elementary and useful way to construct 1-forms on a surface of genus 2 from 1-forms defined on tori, given by [Far]. A similar construction is made in [Im2].

Start by taking two smooth and closed 1-forms: $\omega_{1}$, defined on a torus $T_{1}$ with periods $c_{1}, d_{1}$, and $\omega_{2}$, defined on a torus $T_{2}$, with periods $c_{2}, d_{2}$.

We will take the connected sum of the two tori and define a 1-form $b$ on the bitorus. In order to do this, in each tori we consider sufficiently small open sets $N_{1}$ and $N_{2}$ which are neighborhoods of regular points. On each of them the 1-form is written as $d z$, where $(y, z)$ are the coordinates on $\mathbb{R}^{2}$.

We also define the Morse 1 -form $d z$ on $T$, a cylinder diffeomorphic to $\mathbb{S}^{1} \times(0,1)$, embedded in $\mathbb{R}^{3}$ with coordinates $(x, y, z)$, whereby we form the sum. A homotopically non-trivial simple closed curve in $T$ will be in the collar of the resulting bitorus.

Remove an open disk $D_{1}$ inside $N_{1}$ and identify an open cylindrical neighborhood of $\partial D_{1}$, restricted to $N_{1} \backslash D_{1}$, to an open cylindrical neighborhood of $\mathbb{S}^{1} \times\{0\}$ in $T$. Do the same with an open disk $D_{2}$ inside $N_{2}$ and $\mathbb{S}^{1} \times\{1\}$.

The foliations will be assembled, and we may write

$$
\left.b\right|_{T}=d z,\left.b\right|_{T_{k}-N_{k}}=\omega_{k}, \text { for } k=1,2 .
$$


The result is a closed 1 -form $b$ with periods $c_{1}, d_{1}, c_{2}, d_{2}$.

Now, we shall describe separately four model cases, the last one obtained by a slightly different construction. The position of $T$ determines the interaction of the foliations on the tori, and in all cases two saddle points appear in $T$ where $d z$ is equal to 0 , which is where the tangent plane to $T$ is horizontal.

Besides the connected sum, we can also form an operation called attaching handles. In this case we build the 1-form on a bitorus by using two disjoint neighborhoods of a same torus and connecting them by means of the model cases below. We will give examples of this in Chapter 5.

\subsubsection{Case A}

In Case $\mathrm{A}$, the two singular points in $T$ are connected by two compactifiable leaves. In Figure 3.1 we depict some leaves of $b$, which are level sets of a local primitive of $b$.
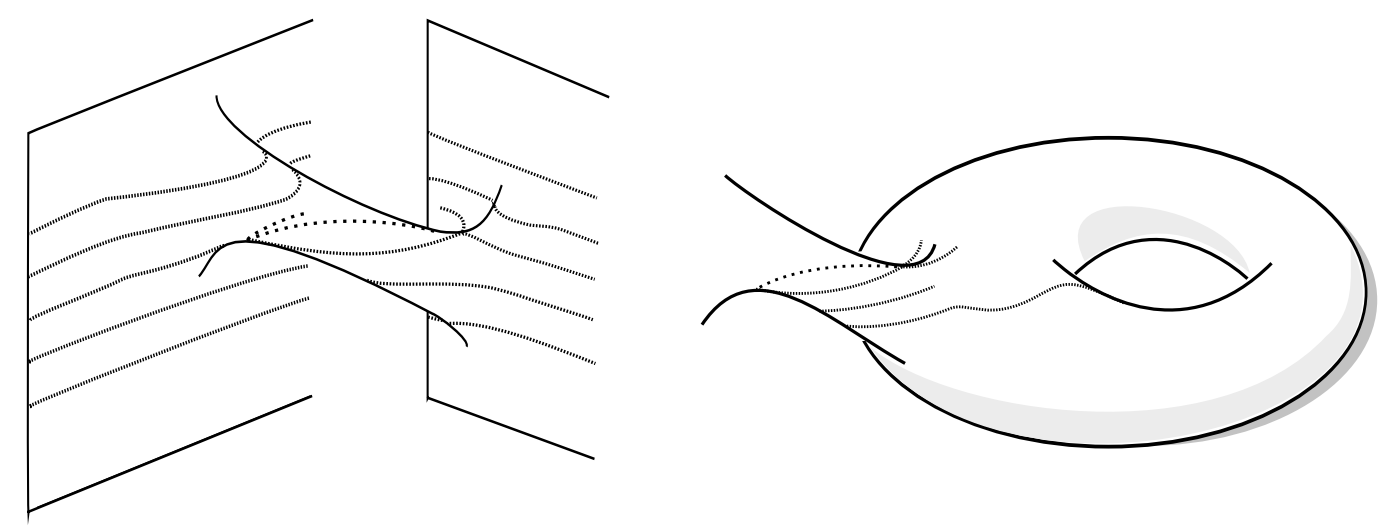

Figure 3.1: Case A

We will look at what happens in a covering space of the surfaces $M_{k}$, with boundary, obtained by cutting our surface along the simple closed curve containing the compactifiable leaves in the collar. Call $M_{k}^{\prime}$ an open subset of $M$ equal to the union of $M_{k}$ and a small cylindrical neighborhood $T^{\prime} \subset T$ of this cut, which is homeomorphic to $\mathbb{S}^{1}$.

Consider a cylinder (or a plane) with open holes that covers $M_{k}^{\prime}$.

On it, it is possible to define a pseudoperiodic function $B_{k}$ by integrating the pullback of $\left.b\right|_{M_{k}^{\prime}}$ (the integral along simple closed curves is zero).

Call $\widetilde{M}_{k}$ the subset of this covering that covers $M_{k}$ and let us study $B_{k}$ on it.

We reserve the term pipe for each piece of $T$ after the cut (each one being diffeomorphic to $\mathbb{S}^{1} \times[0,1)$ ), and we say that $M_{k}$ is a torus $T_{k}$ to which a pipe has been attached. 


\subsubsection{Case B}

Case B is similar to Case A, but this time there is a maximal component between the compactifiable leaves (see Figure 3.2).
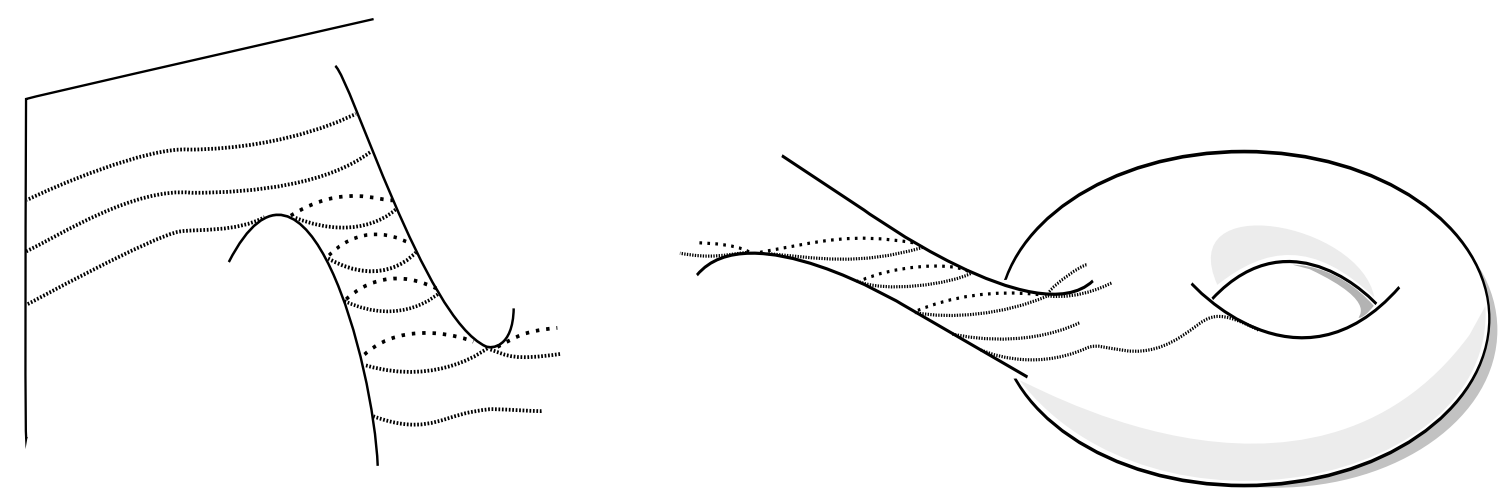

Figure 3.2: Case B

We will cut along the two $\mathbb{S}^{1}$ containing the compactifiable leaves in the collar, and now we produce three pieces by cutting $T$, two of them are pipes with a configuration as in Case A, and the other one is an open cylinder.

\subsubsection{Case $\mathrm{C}$}

In this case, we consider a vertical $\mathbb{S}^{1}$ in the collar containing both critical points (see Figure 3.3).

Observe that, since $\left.b\right|_{T}=d z$, one saddle is the point of maximum of the local primitives restricted to this $\mathbb{S}^{1}$, and the other is the point of minimum. These points determine two arcs, and the intermediate values are attained only once on each such arc. The cut is made along this closed curve.

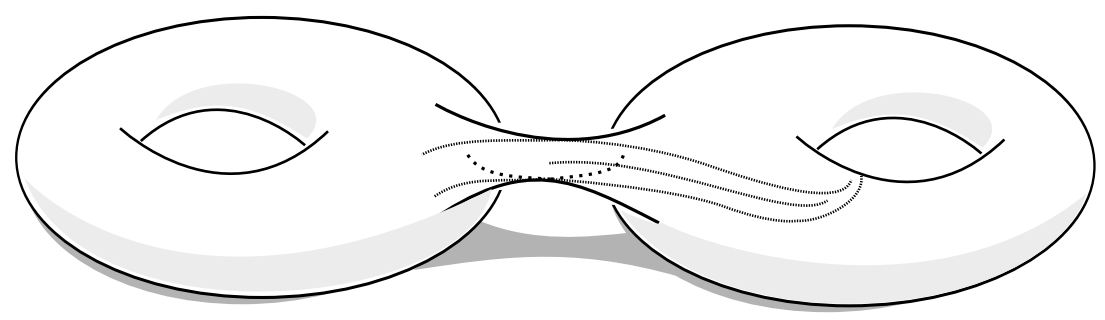

Figure 3.3: Case C

\subsubsection{Case D}

Here the connected sum is such that in $T$ there will be the foliation given by the level sets of the function $f(x, y)=\sin (x) \sin (y)$ defined on the cylinder $(-\pi / 2, \pi / 2) \times \mathbb{S}^{1}$ 
(in Figure 3.4 we show the levels of $f$ viewed as defined on $(-\pi, \pi) \times(-\pi, \pi))$. Notice this foliation is also consistent with $d z$ defined on the open sets $N_{1}$ and $N_{2}$, and we will have two saddle points in $T$.
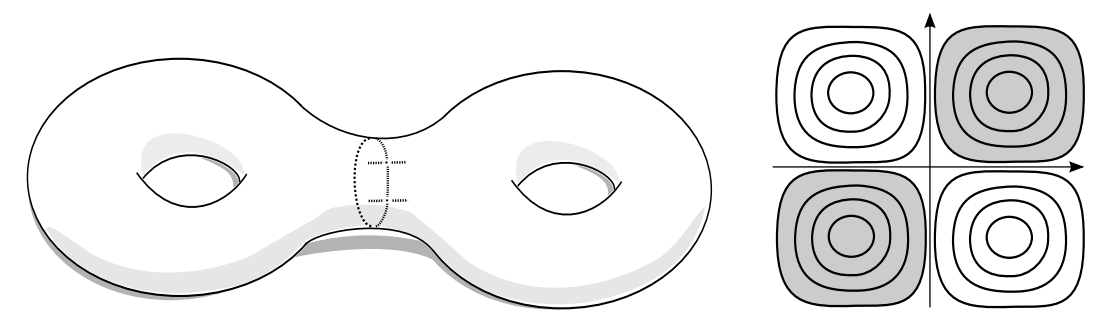

Figure 3.4: Case D

Again, we cut along the $\mathbb{S}^{1}$ containing the compactifiable leaves in the collar. Some examples of smooth 1-forms constructed by this method appear in the examples of Figures 5.2 and 5.3.

\subsection{Our class of examples and auxiliary results}

At first, we shall see that it is possible to iterate the above process of connecting surfaces or attaching handles.

Consider $l \leqslant g$ tori on each of which a closed 1-form $\omega_{k}$ with rank greater or equal to 1 is defined, for $k=1, \ldots, l$. Assume that the primitives of each $\omega_{k}$ have connected sublevels and superlevels in its respective minimal covering space. In these tori one selects disjoint neighborhoods of regular points. We obtain a surface $M$ of genus $g$ and a closed 1 -form $b$ by connecting pairwise these neighborhoods as in the above model cases.

In Figure 3.5 we give an example of this.

We define $M_{k}, k=1, \ldots, l$, by making the cuts and we have that $M_{k}$ is covered by $\widetilde{M}_{k}$, a cylinder (or a plane) with possibly more than one boundary component in each fundamental domain. Moreover, on $\widetilde{M}_{k}$ a pseudoperiodic function $B_{k}$ is defined.

Our goal in this chapter will be to prove the solvability of $\mathbb{L}$ for this class of examples.

Theorem 3.6. The system defined by $b$ is globally solvable in the sense of Definition 1.3.

The crucial point here for the solvability is that we can adapt the proof in [BK]. We see from the above figures the good behaviour of the level sets of the pipes in each covering space, and it turns out that 


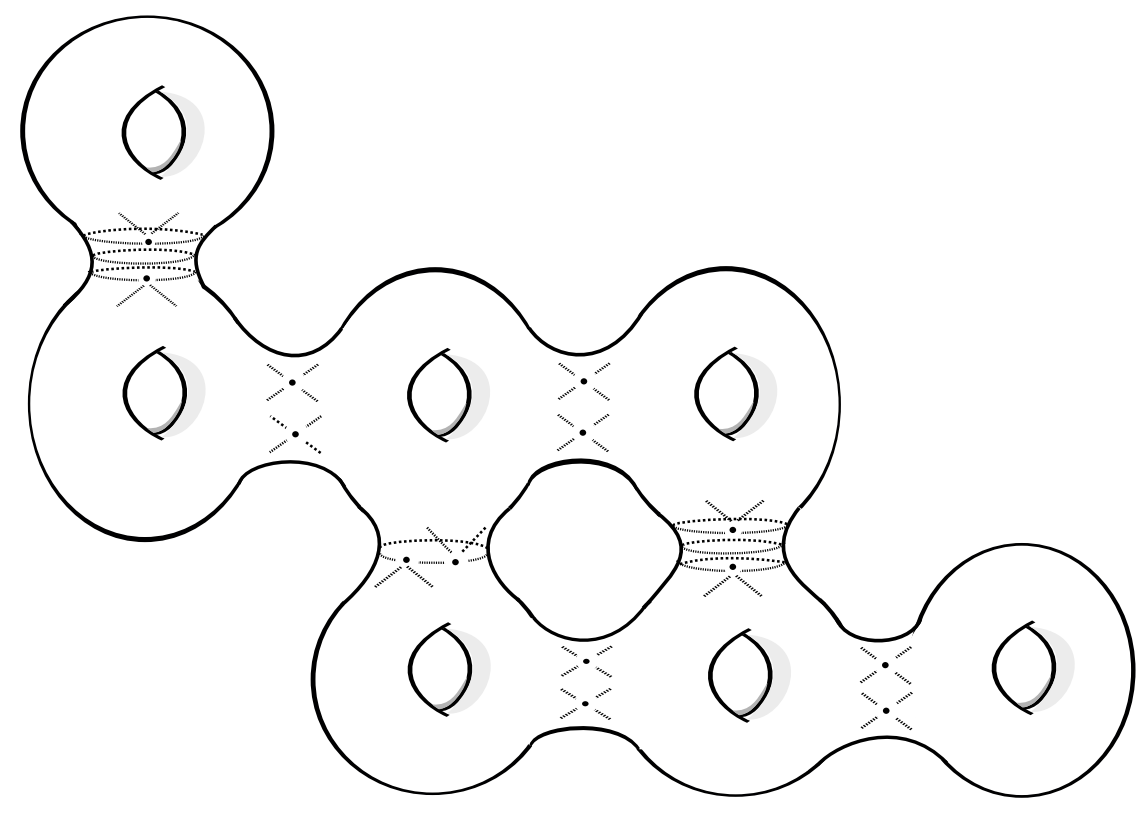

Figure 3.5: Higher genus

Claim. $\Omega_{r}=\left\{\tau \in \widetilde{M_{k}}: B_{k}(\tau)<r\right\}$ and $\Omega^{r}=\left\{\tau \in \widetilde{M_{k}}: B_{k}(\tau)>r\right\}$ are connected, for every $r \in \mathbb{R}$.

Proof. In fact, we have that $B_{k}$ obtained by integration in $\widetilde{M}_{k}$ is equal to a former primitive of $\omega_{k}$ out of open disks, which contain the holes and are inside a fundamental domain in the cylinder or in the plane. Now, fix $r$ and take two points both in $\Omega_{r}$ or both in $\Omega^{r}$. For a path $\alpha$ connecting these two points that intersects such a disk $D$, take $s_{1}=\inf \{s \in[0,1]: \alpha(s) \in D\}$ and $s_{2}=\sup \{s \in[0,1]: \alpha(s) \in D\}$. Since $\omega_{k}$ corresponds to $d z$ on $\Pi(D)$, we can easily replace $\alpha\left(\left[s_{1}, s_{2}\right]\right)$, by an arc in $\partial D$ connecting $\alpha\left(s_{1}\right)$ and $\alpha\left(s_{2}\right)$ in $\Omega_{r}\left(\right.$ or $\left.\Omega^{r}\right)$.
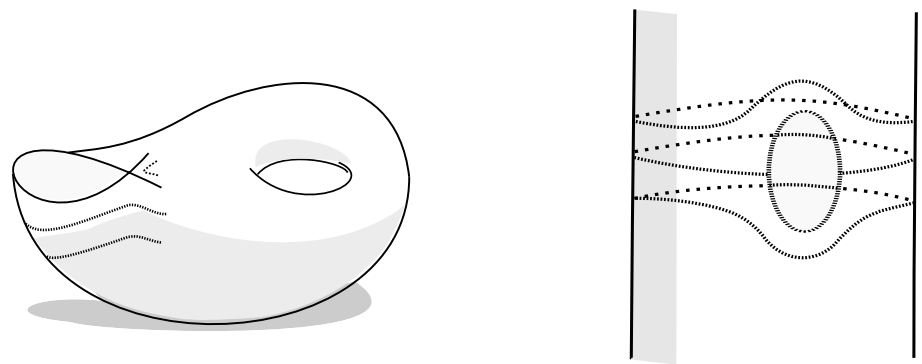

Figure 3.6: Surfaces with pipes attached

From now on, fix $k \in\{1, \ldots, l\}$. We divide our approach according to the rank of $\omega_{k}$. 


\subsubsection{Incommensurable case}

Our fundamental domain will be the square $[0,2 \pi] \times[0,2 \pi]$ with one or more disjoint $\mathbb{S}^{1}$ in $(0,2 \pi) \times(0,2 \pi)$ as boundary components.

We can extend $B_{k}$ to a smooth function on the plane and apply Theorem 4.30 from $[\mathrm{BK}]$ in order to find coordinates in a such way that we can consider the verticals as transversal lines: lines along which $B_{k}$ is strictly monotonic.

When we are dealing with Cases A, B and D, a transversal line does not intersect a boundary component of $\widetilde{M}_{k}$. Still, in Case C, one could have that. Therefore, we take $L$ to be the union of the set $[-2 \pi, 4 \pi] \cap \widetilde{M}_{k}$ and the boundary components that it intersects.

Here we have $B_{k}(t+(2 \pi, 0))=B_{k}(t)+c_{k}$ and $B_{k}(t+(0,2 \pi))=B_{k}(t)+d_{k}$, for $t \in \mathbb{R}^{2}$. Since any pseudoperiodic funtion on the plane is a sum of a periodic function with a linear function, we may consider a non-vertical strip $S_{1}=\left\{\tau=\left(\tau_{1}, \tau_{2}\right) \in \mathbb{R}^{2}\right.$ : $\left.\left|c_{k} \tau_{1}+d_{k} \tau_{2}\right| \leqslant \nu\right\}$ containing all the levels of the points in $[-2 \pi, 4 \pi] \times[0,2 \pi]$. Again, we set $S$ for the union of $S_{1} \cap \widetilde{M}_{k}$ and the boundary components that it intersects.

Lemma 3.7. If $\Omega_{r}=\left\{\tau \in \widetilde{M}_{k}: B_{k}(\tau)<r\right\}$ and $\Omega^{r}=\left\{\tau \in \widetilde{M}_{k}: B_{k}(\tau)>r\right\}$ are connected, for every $r \in \mathbb{R}$, then the sets $K_{r}=\Omega_{r} \cap L \cap S$ and $K^{r}=\Omega^{r} \cap L \cap S$ are bounded and path-connected.

Proof. Since $\Omega_{r}$ is connected, take a path $\alpha$ connecting two of its points and suppose that $\alpha$ is not contained in $L$. Call $P_{1}=\alpha\left(s_{1}\right)$ and $P_{2}=\alpha\left(s_{2}\right)$ respectively the first and the last point of $\alpha$ that intersects a same component of $\partial L$. We replace $\alpha\left(\left[s_{1}, s_{2}\right]\right)$ by the vertical segment $\left[P_{1}, P_{2}\right]$ if a construction as in Case $\mathrm{C}$ is not present.

Otherwise, a vertical segment can intersect a boundary component, say in $Q_{1}$ and $Q_{2}$ such that $B_{k}\left(Q_{1}\right)<B_{k}\left(Q_{2}\right)$, and we use the arc of the $\mathbb{S}^{1}$ connecting them on which $B_{k}$ is less than $B_{k}\left(Q_{2}\right)$ in order to replace $\alpha\left(\left[s_{1}, s_{2}\right]\right)$. In all cases, the chosen path is still in $\Omega_{r}$.

As for $S$, if the modified $\alpha$ is not contained on it, call $P_{1}=\alpha\left(s_{1}\right)$ and $P_{2}=\alpha\left(s_{2}\right)$ respectively the first and the last point of $\alpha$ that intersects a same component of $\partial S$. Again replace $\alpha\left(\left[s_{1}, s_{2}\right]\right)$ by using segments in one of the lines $c_{k} \tau_{1}+d_{k} \tau_{2}= \pm \nu$ and arcs of the boundary components that the line intersects, on which $B_{k}$ is less than $r$; this new path is still in $L$.

A similar proof is valid for the superlevels.

One can compute the Fourier coefficients of the candidate to the problem's solution on $\widetilde{M}_{k}$ by solving a differential equation as in (1.5) for each $\xi \in \mathbb{Z}$, which yields 


$$
\widehat{u_{k}}(t, \xi)=\int_{t_{0}}^{t} v_{k}+K_{\xi} e^{\xi B_{k}(t)},
$$

where $v_{k}(s)=e^{-\xi\left[B_{k}(s)-B_{k}(t)\right]} \hat{f}(s, \xi)$.

Now, if we impose the periodicity in order to define a solution on $M_{k}$, we uniquely determine $K_{\xi}$ and the coefficients of such a solution, namely

$$
\widehat{u_{k}}(t, \xi)=\rho_{k}(\xi) \int_{t}^{t+(2 \pi, 0)} v_{k},
$$

where $\rho_{k}(\xi)=\left(e^{-\xi c_{k}}-1\right)^{-1}$.

On the other hand, we also have

$$
\widehat{u_{k}}(t, \xi)=\rho_{k}(-\xi) \int_{t}^{t-(2 \pi, 0)} v_{k} .
$$

Provided that the sets $\Omega_{r}$ and in $\Omega^{r}$ are connected, we construct some paths that will enable us to conclude the decay of the Fourier coefficients.

Proposition 3.8. For each $\xi \in \mathbb{Z}$ and $t$ in a fundamental domain there exist paths $\gamma(t, \xi)$ in $\widetilde{M}_{k}$, connecting $t$ to $t+(2 \pi, 0)$ or $t-(2 \pi, 0)$, with the following properties:

(i) $B_{k}(\tau) \leqslant B_{k}(t)+\frac{1}{1+|\xi|}, \quad \tau \in \gamma(t, \xi) ;$

(ii) $|\gamma(t, \xi)| \leqslant C(1+|\xi|)$.

Proof. We have to consider the levels $r=B_{k}(t)+\frac{1}{2(1+|\xi|)}$ and the intersection of the plane squares with side-length $2^{-j} 2 \pi$ with $\widetilde{M}_{k}$. If we set $\vartheta_{j r}$ for the collection of these squares (we could have subsets of some squares) that have intersection with $K_{r}$, then the result is a bounded and connected set.

Let us connect $t$ to $t+(2 \pi, 0)$ if $c_{k}<0$ (or to $t-(2 \pi, 0)$ if $c_{k}>0$ ) by a path $\alpha$ in $\vartheta_{j r}$. The path $\gamma(t, \xi)$ will be a piecewise linear path obtained by modifying $\alpha$ as follows. In each square $Q$ of $\vartheta_{j r}$, the path $\alpha$ has initial and final points, which, by considering $j$ big enough, can be connected by at the most two segments inside $Q$ and avoiding the boundary components.

Now, if $s$ is a point of these segments, and $s^{\prime}$ is the initial point (or the final point, depending on $s$ ), since the diagonal of $Q$ has length $\pi \sqrt{2} / 2^{j-1}$, we have

$$
B_{k}(s) \leqslant\left|B_{k}(s)-B_{k}\left(s^{\prime}\right)\right|+B_{k}\left(s^{\prime}\right) \leqslant\|b\|_{\infty} \pi \sqrt{2} / 2^{j-1}+r .
$$

Choose $j$ such that 


$$
2^{j-1} \leqslant 4 \pi \sqrt{2}(1+|\xi|)\|b\|_{\infty} \leqslant 2^{j}
$$

The right inequality of (3.3) allows us to conclude the estimate $(i)$.

If $N$ is the number of fundamental domains that intersect $K_{r}$, the number of elements in $\vartheta_{j r}$ is at most $4^{j} N$, and hence the length

$$
|\gamma(t, \xi)| \leqslant 2.4^{j} N \cdot \pi \sqrt{2} / 2^{j-1}
$$

In possession of the left inequality in (3.3), we are able to conclude the estimate (ii). At the end, we locally modify $\gamma(t, \xi)$ in order to make it smooth, but without affecting the estimates.

Similarly, we have

Proposition 3.9. For each $\xi \in \mathbb{Z}$ and $t$ in a fundamental domain there exist paths $\gamma(t, \xi)$ in $\widetilde{M}_{k}$, connecting $t$ to $t+(2 \pi, 0)$ or $t-(2 \pi, 0)$, with the following properties:

(i) $B_{k}(\tau) \geqslant B_{k}(t)-\frac{1}{1+|\xi|}, \quad \tau \in \gamma(t, \xi)$;

(ii) $|\gamma(t, \xi)| \leqslant C(1+|\xi|)$.

\subsubsection{Lower rank}

We will follow the steps of the previous section.

Notice that we can extend $B_{k}$ to a smooth function on the cylinder such that $B_{k}(t+(0,2 \pi))=B_{k}(t)+d_{k}^{\prime}$, with $d_{k}^{\prime} \neq 0$, for $t \in \mathbb{S}^{1} \times \mathbb{R}$ and $(0,2 \pi) \in \mathbb{R}^{2} \times \mathbb{R}$.

Consider $S=\left\{\tau=\left(\tau_{1}, \tau_{2}\right) \in \mathbb{R}^{2} \times \mathbb{R}:\left|d_{k}^{\prime} \tau_{2}\right| \leqslant \nu\right\}$ a horizontal strip containing all the levels of the points in $\mathbb{S}^{1} \times[0,2 \pi]$. Without loss of generality, we can choose this strip not intersecting a boundary component of $\widetilde{M}_{k}$.

Lemma 3.10. If $\Omega_{r}=\left\{\tau \in \widetilde{M}_{k}: B_{k}(\tau)<r\right\}$ and $\Omega^{r}=\left\{\tau \in \widetilde{M_{k}}: B_{k}(\tau)>r\right\}$ are connected for every $r \in \mathbb{R}$, then the sets $K_{r}=\Omega_{r} \cap S$ and $K^{r}=\Omega^{r} \cap S$ are bounded and path-connected.

The proof for this case is analogous. Actually, the situation became simpler since $S$ is bounded, thus we do not need to be concerned about considering the transversal lines.

In Figures 3.7 and 3.8 we illustrate some examples.

One can compute the Fourier coefficients of the candidate to the problem's solution on $\widetilde{M}_{k}$ by solving a differential equation for each $\xi \in \mathbb{Z}$, which yields 

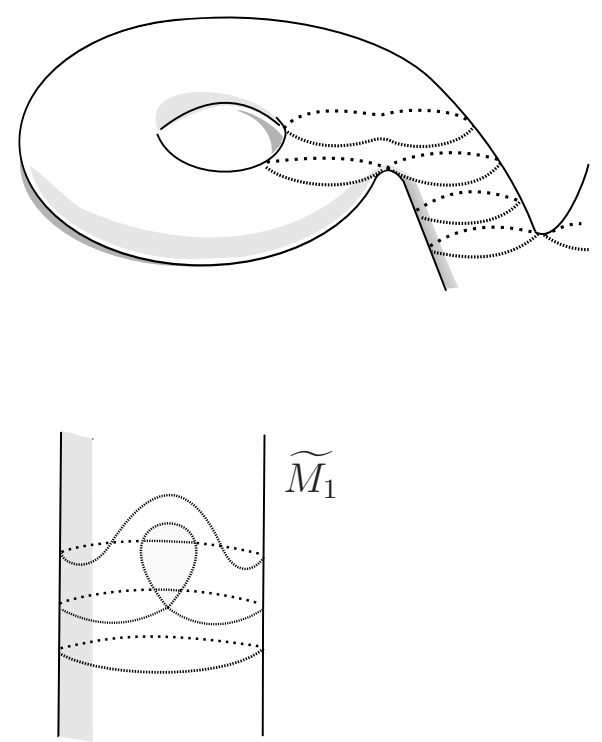

Figure 3.7: Position B and $\omega_{1}=d \theta$

$$
\widehat{u_{k}}(t, \xi)=\int_{t_{0}}^{t} v_{k}+K_{\xi} e^{\xi B_{k}(t)},
$$

where $v_{k}=e^{-\xi\left[B_{k}(s)-B_{k}(t)\right]} \hat{f}(s, \xi)$.

Now, if we impose the periodicity in order to define a solution on $M_{k}$, we uniquely determine $K_{\xi}$ and the coefficients of such a solution, namely

$$
\widehat{u_{k}}(t, \xi)=\rho_{k}(\xi) \int_{t}^{t+(0,2 \pi)} v_{k},
$$

where $\rho_{k}(\xi)=\left(e^{-\xi d_{k}^{\prime}}-1\right)^{-1}$.

Again, we also have

$$
\widehat{u_{k}}(t, \xi)=\rho_{k}(-\xi) \int_{t}^{t-(0,2 \pi)} v_{k} .
$$

By slightly adaptations of Propositions 3.8 and 3.9, one correspondingly has

Proposition 3.11. For each $\xi \in \mathbb{Z}$ and $t$ in a fundamental domain there exist paths $\gamma(t, \xi)$ in $\widetilde{M}_{k}$, connecting $t$ to $t+(0,2 \pi)$ or $t-(0,2 \pi)$, with the following properties:

(i) $B_{k}(\tau) \leqslant B_{k}(t)+\frac{1}{1+|\xi|}, \quad \tau \in \gamma(t, \xi)$;

(ii) $|\gamma(t, \xi)| \leqslant C(1+|\xi|)$. 

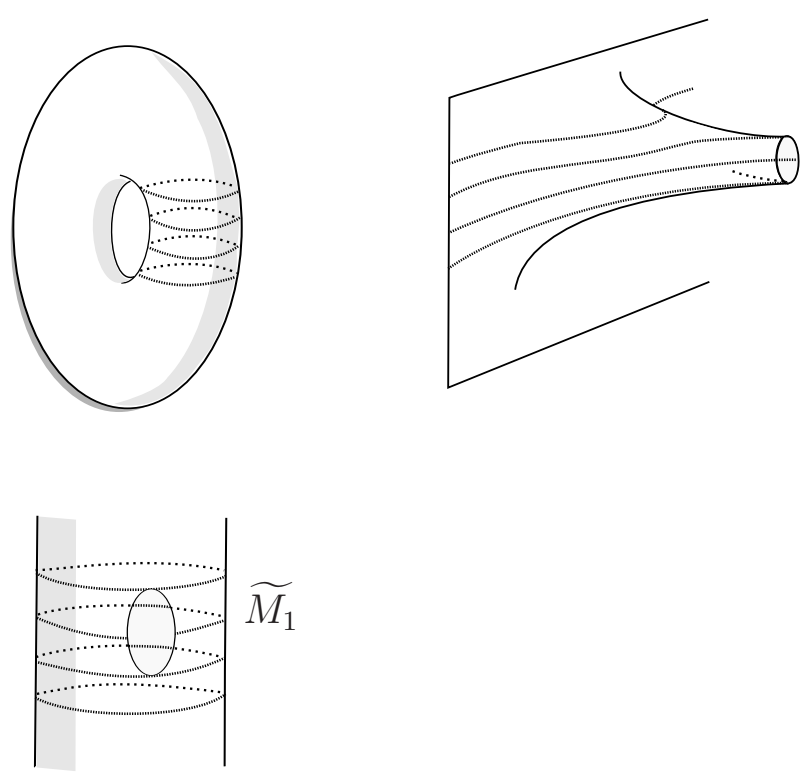

Figure 3.8: Position $\mathrm{C}$ and $\omega_{1}=d \theta$

Proposition 3.12. For each $\xi \in \mathbb{Z}$ and $t$ in a fundamental domain there exist paths $\gamma(t, \xi)$ in $\widetilde{M}_{k}$, connecting $t$ to $t+(0,2 \pi)$ or $t-(0,2 \pi)$, with the following properties:

(i) $B_{k}(\tau) \geqslant B_{k}(t)-\frac{1}{1+|\xi|}, \quad \tau \in \gamma(t, \xi)$;

(ii) $|\gamma(t, \xi)| \leqslant C(1+|\xi|)$.

\subsection{Proof of the global solvability}

Lemma 3.13. If $c_{k}<0$, there exists $C_{0}>0$ satisfying $C_{0}^{-1} \leqslant\left|e^{\xi c_{k}}-1\right| \leqslant C_{0}$, for every $\xi \in \mathbb{Z}^{+}$.

Proof. As $\xi c_{k} \leqslant c_{k}$, we have

$$
\left|1-e^{\xi c_{k}}\right|=1-e^{\xi c_{k}} \geqslant 1-e^{c_{k}} .
$$

Also notice that

$$
\left|1-e^{\xi c_{k}}\right| \leqslant 1+e^{\xi c_{k}} \leqslant 1+e^{c_{k}}<\left(1-e^{c_{k}}\right)^{-1}
$$

since $\left(1+e^{c_{k}}\right)\left(1-e^{c_{k}}\right)=1-e^{2 c_{k}}<1$ and $1-e^{c_{k}}>0$.

Notice that $\widetilde{M}_{k}$ is a normal covering space of $M_{k}$. By Proposition 1.38 from [Ha], $\widetilde{M}_{k}$ is isomorphic to a connected set of the minimal covering space $\widetilde{M}$, that covers $M_{k}$ and there $B_{k}$ is equal to $B$, up to a constant. As $f \in \mathbb{E}$, each integral that defines the Fourier coefficients does not depend on the path. 
If the periods are incommensurable, by using the path of Proposition 3.8 in (3.1) or (3.2), we conclude in any case, for $\xi<0$, that

$$
\left|\widehat{u_{k}}(t, \xi)\right| \leqslant C^{\prime}(1+|\xi|) e^{\frac{|\xi|}{1+\xi \mid}} \sup \{|\hat{f}(\tau, \xi)|: \tau \in \gamma(t, \xi)\}
$$

Therefore, for every $N \in \mathbb{Z}^{+}$there exists $C_{N}^{\prime}>0$ such that

$$
\left|\widehat{u_{k}}(t, \xi)\right| \leqslant \frac{C_{N}^{\prime}}{(1+|\xi|)^{N-1}}
$$

For $\xi>0$, we use the path of Proposition 3.9 in (3.1) or (3.2), which means we have the uniform decay of the Fourier coefficients on $M_{k}$, for every $k=1, \ldots, l$.

If the periods are linearly dependent over $\mathbb{Q}$, we have the same conclusion due to Proposition 3.11 applied to (3.4) or (3.5), for $\xi<0$, and Proposition 3.12 applied to $(3.4)$ or $(3.5)$, for $\xi>0$.

We now would like to glue the solutions together, and we emphasize this in the next result.

In our class of examples, we have surfaces $M_{k}, k=1, \ldots, l$, with boundary being simple closed curves. Recall that these tori can be the same if we are attaching a handle.

We recall that $M_{k}^{\prime}$ is the union of $M_{k}$ with a small cylindrical neighborhood of their respective boundary on $M$, for $k=1, \ldots, l$. Given $M_{k}$ and $M_{j}$, such neighborhoods are identified in order to reobtain $M$. Then, we have the following

Proposition 3.14. With the above notation, suppose that we have defined the Fourier coefficients of candidates to be solution of the systems restricted to $M_{k}^{\prime}$ and to $M_{j}^{\prime}$, with uniform rapid decay in $\xi$ on $M_{k}$ and $M_{j}$. Therefore, after the identification, we have the Fourier coefficients of a candidate to be solution to the system restricted to $M_{k}^{\prime} \cup M_{j}^{\prime}$, with uniform decay on $M_{k} \cup M_{j}$.

Proof. The coefficients on the points of the neighborhood could be defined either as $\widehat{u_{k}}(t, \xi)$ or as $\widehat{u_{j}}(t, \xi)$. We can lift to $\widetilde{M}$ the paths that define the coefficients and evaluate them there. Thanks to Proposition 1.4, $\widehat{u_{k}}(t, \xi)=\widehat{u_{j}}(t, \xi)$ for those points.

Now, we put

$$
u_{k}(t, x)=\sum_{\xi \in \mathbb{Z}} \widehat{u_{k}}(t, \xi) e^{i x \xi}
$$

and we want to show that $u_{k} \in C^{\infty}\left(M_{k}^{\prime} \times \mathbb{S}^{1}\right)$.

Since we have calculated the coefficients by solving

$$
d_{t}\left(\widehat{u_{k}}(t, \xi)\right)-\left.\xi b\right|_{M_{k}^{\prime}}(t) \widehat{u_{k}}(t, \xi)=\left.\hat{f}\right|_{M_{k}^{\prime}}(t, \xi),
$$


in local charts of $M_{k}^{\prime}$ we have

$$
\partial_{i} \widehat{u_{k}}(t, \xi)=\xi \partial_{i} B_{k}(t) \widehat{u_{k}}(t, \xi)+\hat{f}(t, \xi)
$$

for $i=1,2$. We will prove the needed uniform decay for the derivatives by induction on their order. Let us denote the order of a multi-index $\alpha$ by $|\alpha|$.

Suppose that we have $|\alpha| \geqslant 0$ such that for every $N \in \mathbb{Z}^{+}$there is $C_{|\alpha|, N}>0$ with

$$
\max \left\{\left|\partial^{\alpha^{\prime}} \hat{f}(t, \xi)\right|,\left|\partial^{\alpha^{\prime}} \widehat{u_{k}}(t, \xi)\right|\right\} \leqslant \frac{C_{|\alpha|, N}}{(1+|\xi|)^{N}}
$$

for $\left|\alpha^{\prime}\right| \leqslant|\alpha|$ and every $\xi \in \mathbb{Z}$.

If we consider a derivative of order $|\beta|=|\alpha|+1$, then, by (3.6), we have for some $i=1,2$ that

$$
\partial^{\alpha} \partial_{i} \widehat{u_{k}}(t, \xi)=\xi \partial^{\alpha}\left[\partial_{i} B_{k}(t) \widehat{u_{k}}(t, \xi)\right]+\partial^{\alpha} \hat{f}(t, \xi) .
$$

Each term in $\partial^{\alpha}\left[\partial_{i} B_{k}(t) \widehat{u_{k}}(t, \xi)\right]$ is $\partial^{\alpha^{\prime}} \partial_{i} B_{k}(t) \partial^{\alpha^{\prime \prime}} \widehat{u_{k}}(t, \xi)$ for some $\alpha^{\prime}, \alpha^{\prime \prime}$ with $\left|\alpha^{\prime}\right|+\left|\alpha^{\prime \prime}\right|=|\alpha|$, and as $b$ is defined on a compact surface, there is a constant $C_{|\beta|} \geq 0$ such that $\left|\partial^{\beta^{\prime}} B_{k}(t)\right| \leqslant \tilde{C}_{|\beta|}$, for every $t \in M_{k}^{\prime}$ and $0<\left|\beta^{\prime}\right| \leqslant|\beta|$.

Hence, for every $N \in \mathbb{Z}^{+}$and $\xi \in \mathbb{Z}$,

$$
\left|\partial^{\beta} \widehat{u_{k}}(t, \xi)\right| \leqslant|\xi| \tilde{C}_{|\beta|} \frac{C_{|\alpha|, N+1}^{\prime}}{(1+|\xi|)^{N+1}}+\frac{C_{|\alpha|, N+1}}{(1+|\xi|)^{N+1}}
$$

This allows us to conclude the infinite differentiability of $u_{k}$ in $M_{k}^{\prime} \times \mathbb{S}^{1}$.

We also have that $u_{j}$ defines a smooth function on $M_{j}^{\prime} \times \mathbb{S}^{1}$, and it coincides with $u_{k}$ on the identified neighborhoods.

Proof of Theorem 3.6. If a construction as in Case B was not involved in the process, we just have to iterate the previous proposition noticing that the Fourier coefficients $\xi \mapsto \widehat{u_{k}}(t, \xi)$ are also defined on $M_{k}^{\prime}$, for $k=1, \ldots, g$.

We have the decay of the coefficients except for the maximal components, which are foliated cylinders.

For Case B, the same formula of the Fourier coefficients of the solution on the torus $M_{k}$ with boundary in the left side of such a cylinder $C$ holds for a candidate to solution on $M_{k} \cup C$ (evaluated on a covering space of $M_{k} \cup C$ ), but we must prove the decay also on $C$.

In order to do this, this time we write the coefficients as 


$$
\widehat{u_{k}}(t, \xi)=\int_{t_{0}}^{t} v_{k}+\rho_{k}(\xi) \int_{\gamma\left(t_{0}, \xi\right)} v_{k},
$$

where $t_{0} \in M_{k}$ could be chosen, for instance, such that the path connecting $t \in C$ to $t_{0}$ is entirely contained in the cylinder and $B_{k}$ is decreasing along it.

Thus, if $\xi>0$, for every $s$ on this path,

$$
e^{-\xi\left[B_{k}(s)-B_{k}(t)\right]} \leqslant 1
$$

and because the second integral is already estimated, we have the rapid decay for $\xi>0$.

Another solution is calculated on the right torus $M_{j}$ with boundary and, thus, on $M_{j} \cup C$, and Proposition 1.4 guarantees that $\widehat{u_{k}}(t, \xi)=\widehat{u_{j}}(t, \xi)$ on $C$. Therefore, we can also conclude the decay when $\xi<0$ on $C$.

If we now follow as above proposition, we will have a smooth function on $M_{k} \cup$ $C \cup M_{j}$ and, by construction, a solution to the system on $M \times \mathbb{S}^{1}$.

Remark 3.15. When $b$ is Morse, the smoothness of a solution in $\mathscr{D}^{\prime}\left(M \times \mathbb{S}^{1}\right)$ is already guaranteed by $[\mathrm{BCM}]$ since $B$ is an open map at the saddle points.

\subsection{Morse forms}

We finish this chapter of examples focusing on the Morse cases.

Corollary 3.16. For a 1-form b obtained as Theorem 3.6 from Morse 1-forms each one defined on a torus without singular points, the operator $\mathbb{L}$ is globally solvable.

The result of our procedure is a Morse 1 -form with $2 g$ saddle points. Note the number of saddles is coherent with the Poincaré-Hopf Theorem.

The global solvability holds separately for each torus with similar conditions of compatibility, and it turns out that we also have it for the resulting surface. Also, notice that the result holds independently of the rank, the existence of compact leaves, or the final genus.

From Chapter 2 we can infer that if we start with singular points of local maximum or local minimum, we will obtain a non-globally solvable system.

It is interesting to notice that the semiglobal solvability of our system restricted to a sufficiently small neighborhood of a saddle does not hold (with corresponding compatibility conditions - see [Tr2]).

In the next two chapters we intend to enlarge the class of Morse forms for which we are able to prove the global solvability. We will see that we can reduce a substantial number of general Morse forms to the constructions of this chapter. 
In order to do this, it will be useful to consider the following lemma and remark. Essentially, they say that for certain Morse forms we are able to reverse the above process of constructing examples.

Lemma 3.17. Suppose we are given a Morse 1-form $b$ on $M$ with a not homotopically trivial closed curve $\gamma$ consisting of a compactifiable leaf and a singular point p. Consider a sufficiently small tubular neighborhood $U$ of $\gamma$ in $M$. Then one side of $U$ has compact leaves. Moreover, the union of the other side and $\gamma$ is a pipe as in Case A.

Proof. Notice that a primitive $B$ is defined on $U$. We assume that $B(p)=0$.

There is only one saddle point in $\gamma$, and the unique possible orientation for the four separatrices implies that the two of them that are not in $\gamma$, called $F_{p}^{1}$ (arriving at $p$ ) and $F_{p}^{2}$ (issuing from $p$ ), must be in the same side of $U \backslash \gamma$, an open cylindrical set which we call $V$.

Call $V_{1}$ and $V_{2}$ the connected components of $V \backslash\left(F_{p}^{1} \cup F_{p}^{2}\right)$.

If we follow the oriented paths $F_{p}^{1}, \gamma$ and $F_{p}^{2}$, the same arguments of Proposition 3.2 give us an open set $V_{1}^{\prime} \subset V_{1}$ foliated by the regular levels $(-\varepsilon, 0)$ of $B$. Notice that, although we have a singular point now, it has a neighborhood whose intersection with $V_{1}$ are two foliated sets.

Similarly, we have $V_{2}^{\prime} \subset V_{2}$ foliated by the regular levels $(0, \varepsilon)$ of $B$ if we follow $F_{p}^{1}$ and $F_{p}^{2}$.

Therefore, we have that $V_{1} \cup V_{2} \cup F_{p}^{1} \cup F_{p}^{2} \cup \gamma$ is a pipe as in Case A.

Again as in Proposition 3.2, we obtain compact leaves in the other side of $U$.

Remark 3.18. This proof can be modified for some cases in which $\gamma$ consists of compactifiable leaves and two singular points, $p$ and $q$. For instance, when

(i) $F_{p}^{1}$ and $F_{p}^{2}$ are contained in the same side determined by $U \backslash \gamma$, and $F_{q}^{1}$ and $F_{q}^{2}$ are contained in the other side; that is, we are in Case A;

(ii) The leaves $F_{p}^{1}$ and $F_{p}^{2}$ are contained in different sides determined by $U \backslash \gamma$, and so do $F_{q}^{1}$ and $F_{q}^{2}$; that is, we are in Case D.

We will see some examples in Chapter 5. 


\section{CHAPTER 4}

GLOBAL SOLVABILITY FOR THE GENERIC CASE

The intent of this chapter is proving the global solvability for systems defined by a generic closed non-exact 1-form $b$ (see Definition 4.7).

Recall that we are allowed to have only saddle points. Therefore, our first goal in this direction is the following

Theorem 4.1. Suppose that $M$ is a surface of genus $g>1$, and $b$ has only saddles, but not connected by leaves. Then the operator $\mathbb{L}$ is globally solvable.

The proof of this result is in Section 4.1 below. We will apply the next theorem from $[\mathrm{Lev}]$.

Theorem 4.2 (Levitt). For every oriented Morse foliation in a surface of genus $g>1$ with only saddles as critical points, but without leaves connecting them, there exist $3 g-3$ pairwise non-intersecting cycles, transversal to the foliation and splitting the surface into pants. There is exactly only one saddle point in each pant. This structure and the trajectories of the leaves are illustrated in Figure 4.1.

Theorem 4.2 is a fundamental ingredient in the proof of Theorem 4.1 as, recall, the techniques that we are going to use in order to attack the problem of the global solvability of $\mathbb{L}$ are basically contained in the previous chapter, namely we will cut the surface producing some tori with boundary to which we can prove the decay of the Fourier coefficients.

\subsection{Construction of almost transversal curves}

Definition 4.3. An almost transversal curve on $M$ is a simple closed curve $\gamma$ where a primitive $B$ of $b$ is defined such that $\left.B\right|_{\gamma}$ has only two critical points (for instance, as we had in Case C). 

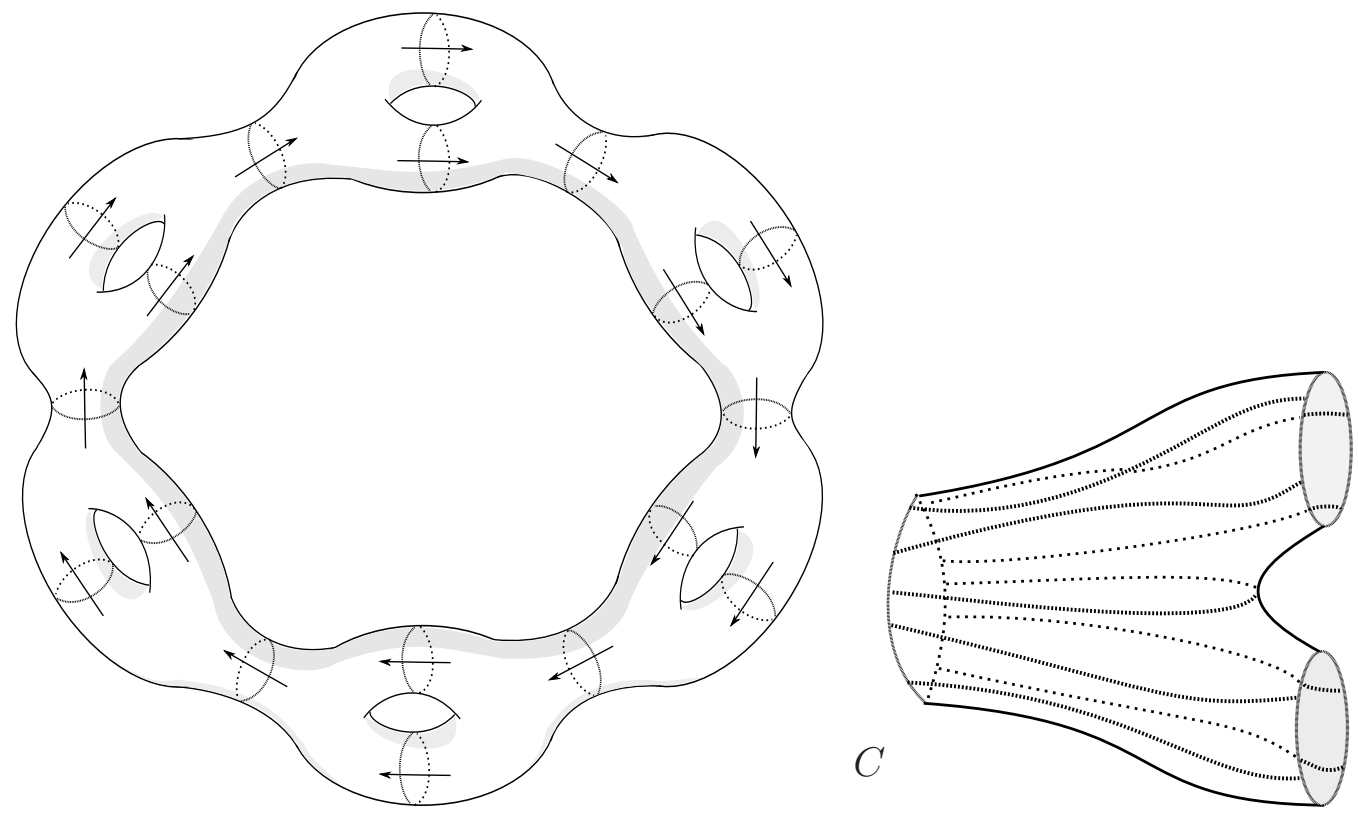

$A$

$B$

Figure 4.1: Decomposition in pants

Remark 4.4. Notice that if $\gamma$ is an almost transversal curve, then in the regular points of $\left.B\right|_{\gamma}$ the foliation is transversal. Hence, $\left.B\right|_{\gamma}$ has a maximum and a minimum value attained exactly once and all intermediate values attained exactly twice.

Also, if the critical points of $\left.B\right|_{\gamma}$ are saddles of $M$, there is a tubular neighborhood $U$ of $\gamma$ in $M$ such that the union of each side of $U$ and $\gamma$ is a pipe as in Case $C$. Indeed, the separatrices of a saddle $p$ in $M$ determine four regions of a sufficiently small neighborhood of $p$, and then $\gamma$ can not intersect two adjacent regions.

We would like to split $M$ by finding some almost transversal curves in collars of $M$.

Begin with two transversal curves, called $C_{1}$ and $C_{2}$, in consecutive pants and of the same type of $C$ in Figure 4.1. We have that $C_{1}$ and $C_{2}$ together cutout a torus called $M_{1}^{*}$ with boundary $\left(\partial M_{1}^{*}=C_{1} \cup C_{2}\right)$.

There are two saddle points in $M_{1}^{*}$, and we call them $p$ and $q$. Consider the leaves, restricted to $M_{1}^{*}$, containing the separatrices (see Figure 4.2). In this section, since such leaves are all different, we use the term separatrix for them. There are two separatrices going from $C_{1}$ to the saddle $p$ and two issuing from the saddle $q$ to $C_{2}$. They determine two disjoint subsets of $M_{1}^{*}$.

In each subset there are other two separatrices. We choose one subset and there we call $F_{p}^{2}$ the separatrix issuing from $p$ to $C_{2}$, and $F_{q}^{1}$ the separatrix going from $C_{1}$ to $q$.

Fix $F_{p}^{1}$ a separatrix from $C_{1}$ to $p$. Notice that in $M_{1}^{*}$ the path $\alpha_{1}$ constructed by following $F_{p}^{1}$ and $F_{p}^{2}$ is free homotopic to the path $\alpha_{2}$ constructed by following $F_{q}^{1}$ 
and one of the separatrices from $q$ to $C_{2}$, depending on the choice of $F_{p}^{1}$.
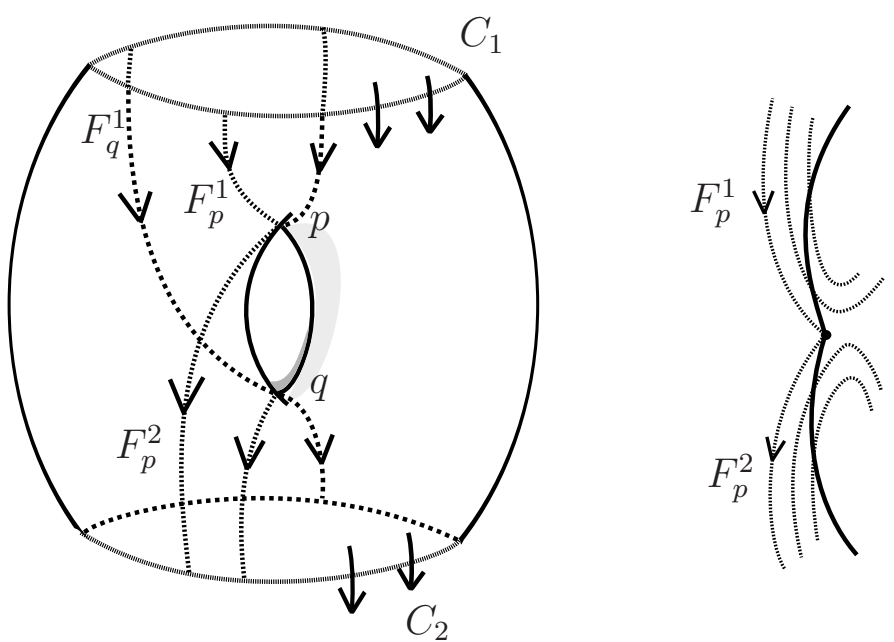

Figure 4.2: The paths $\alpha_{1}$ and $\alpha_{2}$

We now construct a curve $\alpha$ by following $\alpha_{1}$, walking around $C_{2}$, meeting $\alpha_{2}$, walking on it in the opposite direction, walking around $C_{1}$ until meeting $\alpha_{1}$ and closing the path. Since $\alpha_{1}$ and $\alpha_{2}$ are free homotopic in $M_{1}^{*}$, it turns out that $\alpha$ can be constructed dissociating a torus $M_{1}$ with one hole from $M$; thus, it is in a collar of $M$.

The curves $C_{1}$ and $C_{2}$ are transversal to the foliation, hence as we walk around them along $\alpha$ the values of $B$ will increase strictly and decrease strictly (or vice versa).

On the right side of Figure 4.2 the dark line represents a path that is a natural approximation of $\alpha_{1}$, by using a foliated neighborhood of $F_{p}^{1}$ and of $F_{p}^{2}$. We proceed similarly with $\alpha_{2}$ in order to construct an almost transversal curve in the collar, which we keep calling $\alpha$. This procedure of noosing the saddles makes them the critical points of $B$ restricted to $\alpha$, one is a point of maximum and the other one is a point of minimum.

A surface of genus 2 is a sort of special case. There are two pants $P$ and $Q$ containing $p$ and $q$ respectively, and three transversal cycles $A, B$, and $C$. Therefore, $C_{1}$ and $C_{2}$ as above are indeed free homotopic (see Figure 4.3).

This being done, we have constructed $g-1$ almost transversal curves in the collar of a surface of genus $g$ in the case that we do not have saddles connected by leaves, and they split $M$ in $g$ tori with boundary: $g-1$ tori with one hole and one with $g-1$ holes (the central one if $g>2$ - see Figure 4.4).

Now, the global solvability follows from Remark 4.4 and Corollary 3.16. In other words, we have demonstrated that our 1-form $b$ is constructed from Morse 1forms previously defined on tori without singular points, by means of Case $\mathrm{C}$ only. This completes the proof of Theorem 4.1. 

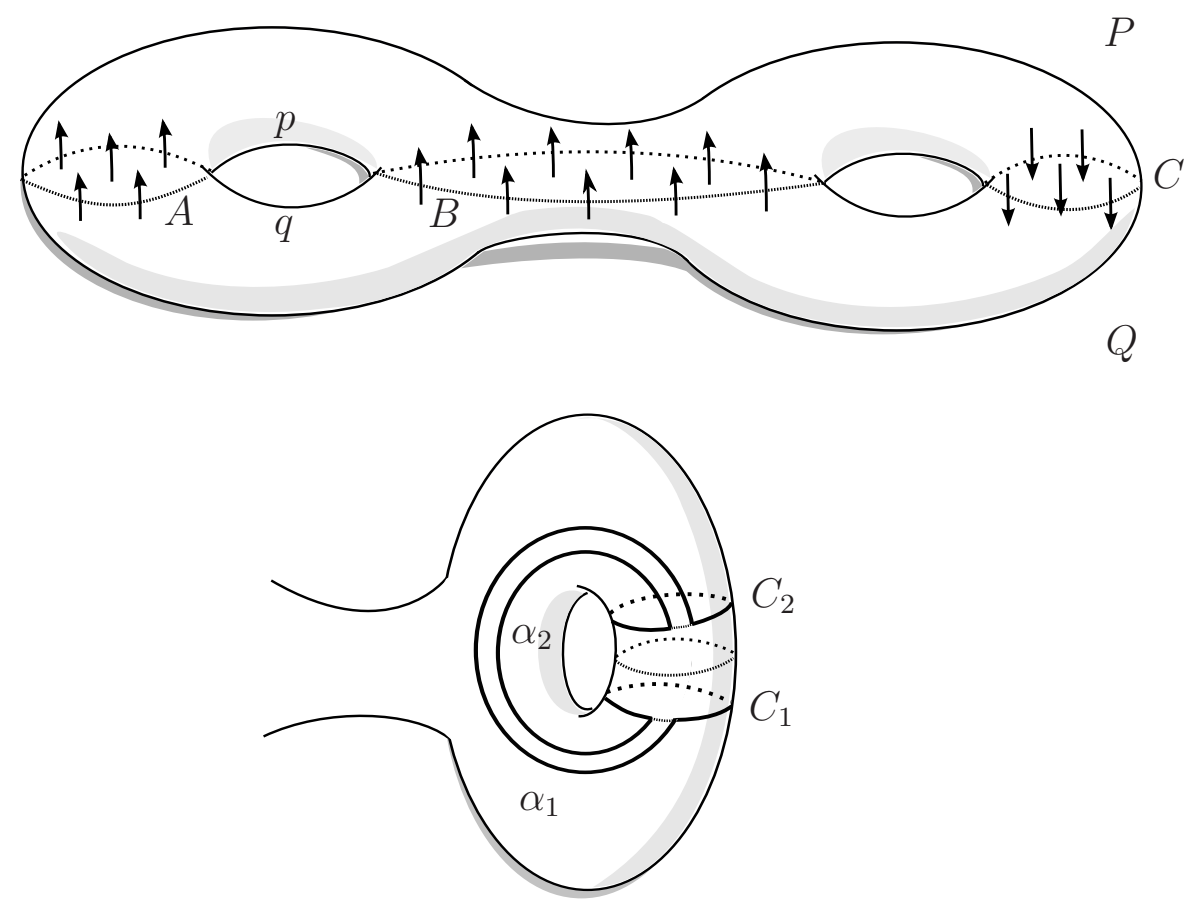

Figure 4.3: Construction of $\alpha$ for genus 2

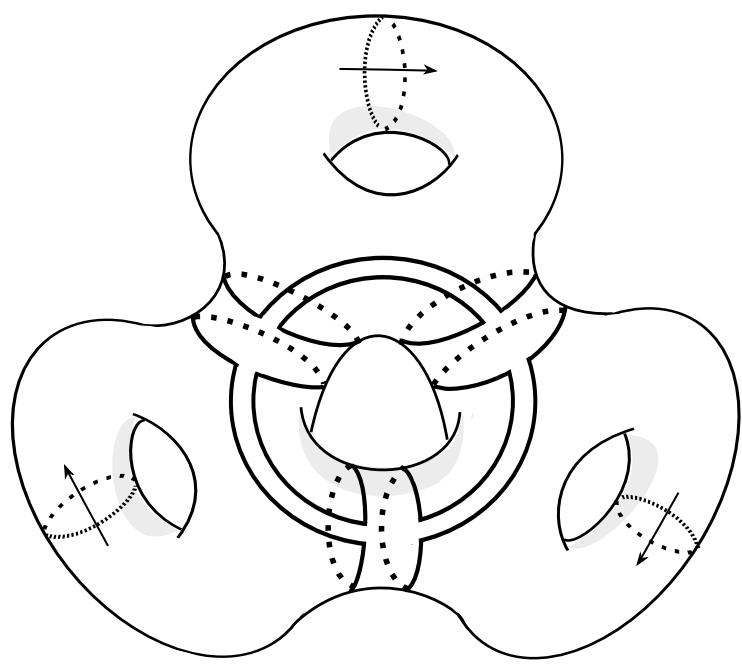

Figure 4.4: Almost transversal curves for genus greater than 2

\subsection{Proof of the generic case}

Example 4.5. Consider $\omega_{1}=r d \theta+s d \phi$, with $r, s \in \mathbb{Q} \backslash\{0\}$, and $\omega_{2}=a \omega_{1}$, with $a \in \mathbb{R} \backslash \mathbb{Q}$, each 1 -form defined on a torus. In each torus the leaves are compact. We carry out the connected sum as in Case C. By increasing the values of $r$ and $s$, it is possible to require that all the leaves of $\omega_{1}$ and $\omega_{2}$ pass through the collar, and hit the other torus. With this construction, the resulting 1-form $b$ does not have either compact leaves or compactifiable leaves. The solution for this example is granted by Corollary 3.16: for each torus with boundary the coefficients are computed on a cylinder since 
on each one the rank is 1 . The minimal covering with respect to b is in Example (iii), Section 1.3.

Example 4.6. This is another example with $\operatorname{rank}(b)=2$. Consider $\omega_{1}=r d \theta+s d \phi$, with $r, s$ linearly independent over $\mathbb{Q}$, and $\omega_{2}=-\omega_{1}$, each 1 -form defined on a torus. Notice that $\omega_{2}$ just reverses the orientation of $\omega_{1}$. We can carry out the connected sum as in Case $C$ such that the result will be a foliation with only compact and compactifiable leaves. A nonsingular leaf will walk around a torus, pass through the collar and do the opposite way in the other torus. In spite of this, again Corollary 3.16 gives us the solution, which this time is computed on two planes with boundary components.

It is nice to see from these examples that the rank does not distinguish topologically different foliations. Therefore, it may not be the best tool to be used in order to decide how we solve the problem.

Another fact is that Theorem 4.1 from [Gel] says that compactifiable foliations may be defined by 1 -forms of different ranks.

As a matter of fact, the theorem by Levitt holds for the first example. It is important to observe that in this theorem there are restrictions on the periods of $b$ (namely those where the transversal cycles are placed are not zero), but no specific mention about the rank.

The theorem by Levitt, however, does not apply when the foliation defined by the 1-form happens to have a simple closed curve consisting of compactifiable leaves and singular points (clearly a case of lower rank when this set is not homologically trivial).

Therefore, we are led to explore now the presence of these compactifiable leaves.

Definition 4.7. A Morse 1-form is called generic when different critical points cannot be connected on $M$ by a leaf.

We now state and conclude the task of this chapter, which extends the result of the previous section.

Theorem 4.8. Suppose we are given a surface $M$ of genus $g>1$ and a generic 1 -form $b$ having only saddle points. Then the operator $\mathbb{L}$ is globally solvable.

Proof. We may assume that we have compactifiable leaves.

First step. Suppose there are minimal components of the foliation. Let us consider the boundary of a minimal component $\mathscr{M}$. We have that $\overline{\mathscr{M}}$ (the closure in $M)$ will be a surface of genus $g^{\prime}, 1 \leqslant g^{\prime} \leqslant g$, whose boundary are disjoint simple closed curves, each one consisting of one compactifiable leaf and one saddle point.

In fact, we are in the situation of Lemma 3.17. Two separatrices of such a saddle are indeed in the same leaf, which is in the boundary of $\mathscr{M}$; the other two separatrices 
cannot be in a same leaf, or else there would be a compact leaf in $\mathscr{M}$. Hence, it is possible to think of $\left.b\right|_{\mathscr{M}}$ as a Morse form $\tilde{b}$ defined on a surface $N$, without boundary, to which some pipes have been attached on small open sets as in Case A.

If $g^{\prime}>1$, there will be $2 g^{\prime}-2$ saddles inside $N$, which are not connected by leaves of $\tilde{b}$. Hence, we apply the procedure of the previous section to $N$, and we can cut the surface. We assume the cuts to be in $\mathscr{M}$ since, if necessary, we can modify them in these open sets (the dark line in Figure 4.5 illustrates this).

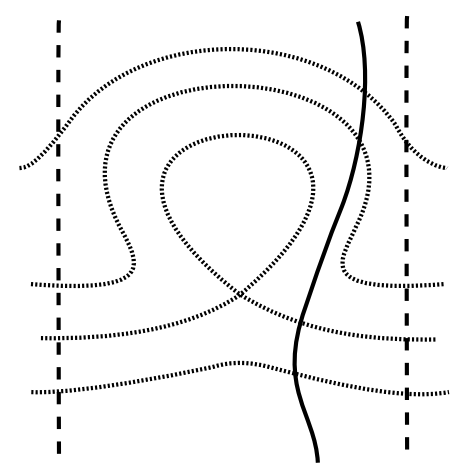

Figure 4.5: Cut avoiding the boundary components

Therefore, in this step we obtain $g^{\prime} \geqslant 1$ tori without singular points to each one of which pipes have been attached as in Cases A or C. As done in the previous chapter, one proves the decay of the Fourier coefficients of a candidate to the solution there.

Second step. If we have compactifiable leaves, then there is a closed curve consisting of a such a leaf and a singular point. By Lemma 3.17, there are also maximal components. Let us remove the minimal components of the foliation and consider $\mathscr{C}$ a component of the resulting surface. Notice that $\mathscr{C}$ is a surface with boundary and consists of finite unions of $\overline{C_{i}}$, with $C_{i}$ being a maximal component.

We will construct a solution inspired by Case B of Theorem 3.6.

Given $t \in \operatorname{int}(\mathscr{C})$, there we find a path called $\beta_{1}$ connecting $t$ to $t_{1}$ in a boundary component such that a primitive is strictly increasing along $\beta_{1}$, and a path called $\beta_{2}$ connecting $t$ to $t_{2}$ in a boundary component such that a primitive is strictly decreasing along $\beta_{2}$.

The formula of the Fourier coefficients of the candidate to solution on the torus $M_{1}$ with boundary obtained in the previous step, with $t_{1} \in M_{1}$, can be extended for a candidate to solution on $M_{1} \cup \mathscr{C}$ (evaluated on a covering space of $M_{1} \cup \mathscr{C}$ ). Using the notation of the previous chapter, on $\mathscr{C}$ the Fourier coefficients are written as

$$
\widehat{u}(t, \xi)=\int_{\beta_{1}} v_{1}+\rho_{1}(\xi) \int_{\gamma_{1}\left(t_{1}, \xi\right)} v_{1} .
$$

Thanks to Proposition 1.4, we can also write 


$$
\widehat{u}(t, \xi)=\int_{\beta_{2}} v_{2}+\rho_{2}(\xi) \int_{\gamma_{2}\left(t_{2}, \xi\right)} v_{2} .
$$

By means of $\beta_{1}$ and $\beta_{2}$, we obtain the decay for $\xi>0$ and for $\xi<0$ respectively. In possession of the rapid decay on every surface with boundary that we have produced, we apply Proposition 3.14. 


\section{CHAPTER 5}

BITORUS REVISITED

We turn our attention again to the surface of genus 2. Let us consider in this chapter the bitorus on which a Morse 1-form $b$ is defined with only two saddle points.

As we have already established the result for the generic case, in order to cover all the possibilities for $b$, we assume in the first section below that the foliation has a simple closed curve called $\gamma$ consisting of compactifiable leaves and two saddle points. There are some examples of this case given above, for instance when we carried out a connected sum as in Case A. We will have to deal with some combinatorics about the position of the separatrices. Even so, for the bitorus, it is possible to give a description for $b$ and also examples.

Afterwards, we will complement it by studying those case in which there is only one compactifiable arc connecting different saddle points.

It will turn out that in all cases $b$ defines globally solvable systems.

\subsection{Case 1}

First of all, suppose two compactifiable arcs and the two saddles, $p$ and $q$, together give a simple closed curve $\gamma$. Consider a sufficiently small tubular neighborhood $U$ of $\gamma$ in $M$

Let us call $F_{p}^{i}$ the separatrices of $p$ not intersecting $\gamma$, and $F_{q}^{i}$ the separatrices of $q$ not intersecting $\gamma, i=1,2$.

The possibilities that may ensue are listed below. Any other combinatorics are forbidden due to the impossibility of giving a consistent orientation for the separatrices.

Case 1.1. $F_{p}^{1}$ and $F_{p}^{2}$ are contained in the same side determined by $U \backslash \gamma$ and $F_{q}^{1}$ and $F_{q}^{2}$ are contained in the other side.

When $\gamma$ is homologically trivial, that is, $\gamma$ is in the collar, we have examples of 
this situation by making a connected sum as in Case A.

If $\gamma$ is not homologically trivial, an example is obtained by attaching a handle to a torus as in Case A (see Figure 5.1). First consider the function $B(x, y)=c_{1} x+d_{1} y$ defined on the plane $x y$, with $c_{1}, d_{1}$ being linearly independent over $\mathbb{Q}$. In a fundamental square, we choose sufficiently small open sets $N_{1}$ and $N_{2}$ to attach pipes as in Case A, and we keep calling $B$ the resulting function. We want to suitably identify the boundary components in a fundamental domain (without identifying a singular point with the other one) so that we can construct a pseudoperiodic function $B^{\sharp}$ on the lollipops.

In order to do this, both boundary components must be in the same critical level set, say $B^{-1}(r)$. Moreover, for $B^{\sharp}$ to be Morse, in one boundary component the compactifiable leaf must in the boundary of the $r$-sublevel of $B$, and in the other boundary component the compactifiable leaf must in the boundary of the $r$-superlevel of $B$.

Clearly, this is a rank 2 example, and there is another compactifiable arc connecting the saddles.

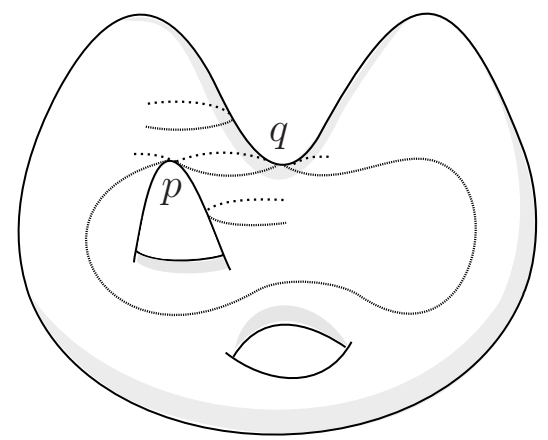

Figure 5.1: Case 1.1

Case 1.2. The leaves $F_{p}^{1}$ and $F_{p}^{2}$ are contained in different sides determined by $U \backslash \gamma$ and so do $F_{q}^{1}$ and $F_{q}^{2}$.

Now, if $\gamma$ is homologically trivial, we obtain examples by making a connected sum as in Case D. Otherwise, if $\gamma$ is homologically non-trivial, Figure 5.2 depicts an example of a foliation which, according to [Gel], can be defined by Morse forms of rank 1 or 2 . For instance, it can be obtained by means of attaching a handle to a torus as in Case D.

Now, notice that due to Remark 3.18 the global solvability in both Case 1.1 and Case 1.2 is taken care by Corollary 3.16.

Indeed, assume that $\gamma$ is homologically trivial and $b$ has both zero periods on one of the tori (called $M_{1}$ ) with boundary, obtained after cutting $M$ along $\gamma$. Then a function $B$ can be defined on $M_{1}$, and we will have a point of global maximum or a 


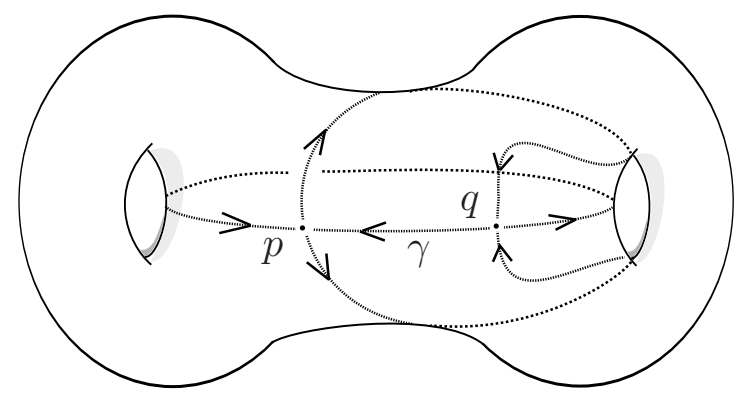

Figure 5.2: Case 1.2

global minimum of $B$ inside it, which is a contradiction.

If the closed curve $\gamma$ is not homologically trivial, we can cut along it obtaining a torus $M_{1}$ with two boundary components, called $\partial D_{1}$ and $\partial D_{2}$, which are copies of $\gamma$. If this torus has both periods zero, we would have that $\operatorname{rank}(b)$ is 1 and we can cover this surface with boundary as it will be done in Chapter 6 below.

Case 1.3. All the leaves $F_{p}^{1}, F_{p}^{2}, F_{q}^{1}, F_{q}^{2}$ are contained in the same side determined by $U \backslash \gamma$.

This situation does not occur when $\gamma$ is homologically trivial. Otherwise, cut along $\gamma$ and consider the resulting torus $M_{1}$ (with boundary $\partial D_{1}$ ) which does not contain the separatrices $F_{p}^{1}, F_{p}^{2}, F_{q}^{1}, F_{q}^{2}$. Then, one could in a natural extend $\left.b\right|_{M_{1}}$ to a Morse 1-form on a torus without boundary.

Remark 5.1. In fact, as in Lemma 3.17, one of the sides $U_{1}$ determined by $U \backslash \gamma$ has compact leaves, and a primitive $B$ of $b$ is defined on $\overline{U_{1}}$. We can extend $B$ to $D_{1}$, and a point of maximum (or minimum) of $\left.B\right|_{D_{1}}$ is created.

Therefore, we would have a Morse 1-form on a torus without boundary and only one singular point, which is a contradiction. Hence, $\gamma$ is homologically non-trivial.

Since one of the sides determined by $U \backslash \gamma$ has compact leaves, we have a maximal component $\mathscr{C}$, whose boundary consists of compactifiable leaves and singular points. This yields that two separatrices not in $\gamma$ are indeed in the same compactifiable leaf.

One of the three remaining regions determined by the separatrices of $p$ in a small open set, namely the one determined by $F_{p}^{1}$ and $F_{p}^{2}$ (called $V_{p}$ ), cannot be in $\mathscr{C}$, for otherwise $b$ would not be Morse (similarly for $q$ and $V_{q}$ ).

The first possibility for the separatrices would be when $F_{p}^{1}$ and $F_{p}^{2}$ are in the same leaf. However, this would imply that there is another maximal component $\mathscr{C}^{\prime}$ such that $F_{p}^{1}$ and $F_{p}^{2}$ are in $\overline{\mathscr{C}^{\prime}}$ and $V_{p} \subset \mathscr{C}^{\prime}$. Now, notice that $F_{q}^{1}$ and $F_{q}^{2}$ must be in $\overline{\mathscr{C}}$, therefore $F_{q}^{1}$ and $F_{q}^{2}$ must also be in a same compactifiable leaf. Hence, $V_{p}$ and $V_{q}$ are in $\mathscr{C}^{\prime}$, which again is not allowed if $b$ is Morse.

The other possibility is when we have that some separatrix of $p$ is in the same leaf of some separatrix of $q$. 
Call $\gamma_{1}$ and $\gamma_{2}$ the compactifiable leaves in $\gamma$. Looking at the boundary of $\mathscr{C}$, we conclude that $p, q$, a compactifiable leaf in $\gamma\left(\right.$ say $\left.\gamma_{1}\right)$, and a compactifiable leaf connecting $p$ to $q$ (called $L$ ) together give a simple closed curve $\delta$ homologous to $\gamma$.

Also, $p, q, L$ and $\gamma_{2}$ give a simple closed curve called $\tilde{\gamma}$. Now,

$$
[\gamma]=[\delta]=[\gamma]+[\tilde{\gamma}]
$$

shows that $\tilde{\gamma}$ is homologically trivial. This implies that we are in a situation already handled above.

We illustrate an example in Figure 5.3 by carrying out a connected sum as in Case D with $\omega_{1}=d \theta$ on $T_{1}$.

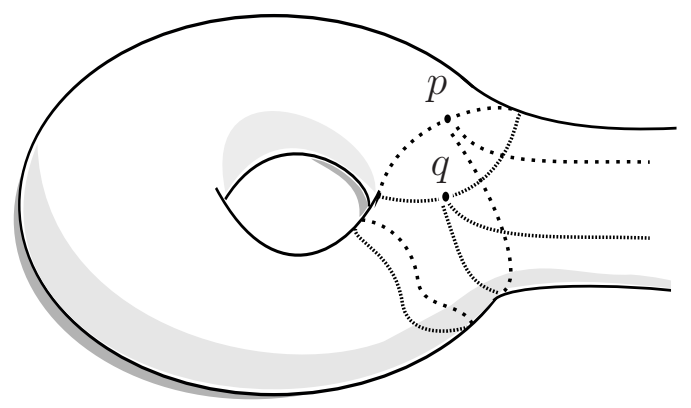

Figure 5.3: Case 1.3

\subsection{Case 2}

We have to consider the cases when there is only one compactifiable arc connecting the two different saddles. Suppose there is still a closed curve $\gamma$ consisting of a saddle, say $p$, and one compactifiable leaf. Due to the orientations that one gives, the separatrices $F_{p}^{1}$ and $F_{p}^{2}$ not in $\gamma$ have to be in the same side determined by $U \backslash \gamma$, where $U$ is a small tubular neighborhood of $\gamma$ in $M$. In one side determined by $U \backslash \gamma$ we have compact leaves, and we call $\mathscr{C}$ their maximal component.

The curve $\gamma$ cannot be in the collar. In fact, if we cut along $\gamma$, we produce a torus $M_{1}$, with boundary, containing these compact leaves and, thus, not containing the other saddle. Hence, as in Remark 5.1, one could construct a Morse form on a torus without boundary by extending $\left.b\right|_{M_{1}}$ and having only one singular point, which is impossible.

Suppose now that the curve $\gamma$ is a homologically non-trivial closed curve. One of the separatrices of $p$ is in a non-compactifiable leaf. This implies that $q \in \overline{\mathscr{C}}$ and there is a closed curve called $\gamma^{\prime}$ consisting of a compactifiable leaf and $q$.

Again, $\gamma^{\prime}$ cannot be in the collar. We claim that, in fact, $\gamma^{\prime}$ is homologous to $\gamma$. Otherwise, by cutting along $\gamma$ one could produce a Morse form on a torus without 
boundary and having a minimal component. However, this minimal component would have less than two independent generators of the homology of the torus, which is a contradiction with Proposition 3.4.

Now, the global solvability is granted by Corollary 3.16. The surface is obtained by attaching a handle as in Case $\mathrm{B}$ to a torus having a non-zero period, after an application of Lemma 3.17.

We give an example constructed by attaching a handle to a torus, similar to the one of Figure 5.1. First consider the function $B(x, y)=c_{1} x+d_{1} y$ defined on the plane $x y$, with $c_{1}, d_{1}$ being linearly independent over $\mathbb{Q}$. In a fundamental square, we choose sufficiently small open sets $N_{1}$ and $N_{2}$ and we attach pipes and cylinders as in Case B. We now want to suitably identify the cylinder issuing from $N_{1}$ with the cylinder issuing from the translate of $N_{2}$ by $(2 \pi, 0)$ so that we can construct a pseudoperiodic Morse function $B^{\sharp}$ on a space as the lollipops. In the end, $d B^{\sharp}$ is defined on the bitorus.

Therefore, for Case 2 it remains to deal with the situation when there is only one compactifiable arc, which is connecting the two saddles (the other leaves are noncompactifiable). We do this in the next section.

\subsection{Construction of an almost transversal curve}

In Chapter 4, we have constructed almost transversal curves for the case when we do not have any leaf connecting the saddles. Now, the strategy will be the same, but we are no longer able to use the strong foliation structure given by Levitt.

As a first step in this direction, we have

Lemma 5.2. Suppose that a foliation $\mathscr{F}$ has a minimal component. Then, there exists a simple closed curve $\beta$ on $M$ transversal to $\mathscr{F}$.

Proof. We consider the vector field $X(t)=\left(-b_{2}(t), b_{1}(t)\right)$, which is tangent to the foliation in $M \backslash \operatorname{sing}(b)$.

By Theorem 3.5, a regular point $A$ in the minimal component is in the $\omega$-limit set of a non-trivial orbit $\varphi$ of this vector field.

In a small foliated neighborhood $V$ of $A$ this orbit passes infinitely many times. Therefore, we can take two points $\varphi\left(s_{1}\right)$ and $\varphi\left(s_{2}\right)$ in $V$, with $s_{1}<s_{2}$, which are not in a same horizontal segment of $V$. We close the orbit by considering the segment in $V$ connecting $\varphi\left(s_{1}\right)$ and $\varphi\left(s_{2}\right)$, which is transversal to the foliation. A natural approximation of $\varphi\left(\left[s_{1}, s_{2}\right]\right)$ is also transversal to the foliation, and we are done.

Now consider the bitorus in the situation of this section; there is only one minimal component in such a foliation. 
Our aim is to place an almost transversal curve in the collar of the surface, and we will adopt a similar construction as in the previous chapter.

For this, call $\beta_{1}$ and $\beta_{2}$ two disjoint cycles homologous to $\beta$ and determining a cylindrical neighborhood $C$ of $\beta$. We can assume, by taking $\beta_{1}$ and $\beta_{2}$ sufficiently close to $\beta$, that they are transversal to the foliation as well. We suppose that the foliation is departing $C$ through $\beta_{1}$ and entering $C$ through $\beta_{2}$.

Since a non-compactifiable leaf is dense in its component, we take an arc $L$, of a non-compactifiable leaf, departing from $\beta_{1}$ until meeting $\beta_{2}$ for the first time.

Take $\alpha_{1}$ and $\alpha_{2}$ two arcs of leaves, both arcs in a small foliated neighborhood of L. Natural approximations of each arc are transversal to the foliation. We keep using the notation $\alpha_{1}$ and $\alpha_{2}$ for these approximations.

Now, consider the curve $\alpha$ by following $\alpha_{1}$, walking around $\beta_{2}$, finding $\alpha_{2}$, walking on it in the opposite direction, walking around $\beta_{1}$ until meeting $\alpha_{1}$, and closing the path so that, at the end, the resulting curve is cobordant to a homotopically non-trivial simple closed curve in the collar. In fact, $\alpha$ can be taken homotopic to a curve of the type $a b a^{-1} b^{-1}$, with $a$ and $b$ being simple closed curves having intersection number equal to \pm 1 .

Moreover, $\alpha$ does not have singular points and is assumed to be smooth. The foliation is tangent to this curve in precisely two points, hence we have constructed an almost transversal curve.

Having this curve, we cut $M$ along it and obtain two tori $M_{1}$ and $M_{2}$, with boundary. Suppose there is a saddle point $p$ inside $M_{1}$. We have

Lemma 5.3. It is possible to noose each saddle point in the covering space of $M_{1}$ by replacing an arc of a boundary component by a path homotopic to it, consisting of the saddle and leaves containing separatrices.

Proof. The rank of $\left.b\right|_{M_{1}}$ is not zero, otherwise we would have a primitive defined on $M_{1}$, and then there will be more separatrices of $p$ lying in compactifiable leaves.

Therefore, the covering space of $M_{1}$ is the cylinder (or the plane) with an $\mathbb{S}^{1}$ as boundary in each respective fundamental domain. We can extend $B$ smoothly to the whole covering space by using a cutoff function.

Consider a saddle $\tilde{p}$ and set $r \doteq B(\tilde{p})$. By following a level set containing a separatrix issuing from $\tilde{p}$, we will meet a boundary component. Due to the configuration of $B$, there is another leaf of the same level set departing the boundary and we can connect these leaves by an arc of this boundary in the $r$-superlevel or in the $r$-sublevel. If we pass through the other singular point of the $r$-level in this procedure, then we choose and follow an adjacent separatrix, in order to keep the orientation.

We claim that there is a separatrix such that, in this way, we come back to $\tilde{p}$ in a homotopically trivial path. 
Indeed, if not, in the case of the plane, the $r$-superlevel or the $r$-sublevel of $B$ would have two unbounded components, which is impossible. Furthermore, we can prove that, after the first boundary component, the level set comes back to $\tilde{p}$ without crossing any other boundary component. Indeed, assume that this level determines a bounded component of, for instance, an $r$-superlevel of $B$ (see Figure 5.4). We consider the first and the last boundary component that this level crosses. If the maximum value of $B$ in one of the boundary components is $K$, then in the other the maximum value is $K+s$, with $s>0$. Now, notice that a saddle $\sigma(\tilde{p})$ (a translate of $\tilde{p})$ such that $B(\sigma(\tilde{p}))=r+s$ is inside this component of superlevel. By repeating this argument, we would have an infinite number of saddles inside the region determined by the homotopically trivial path.

In the case of the cylinder, if we could not come back to $\tilde{p}$ in a homotopically trivial path, again we would have an infinite number of saddles inside a bounded set.

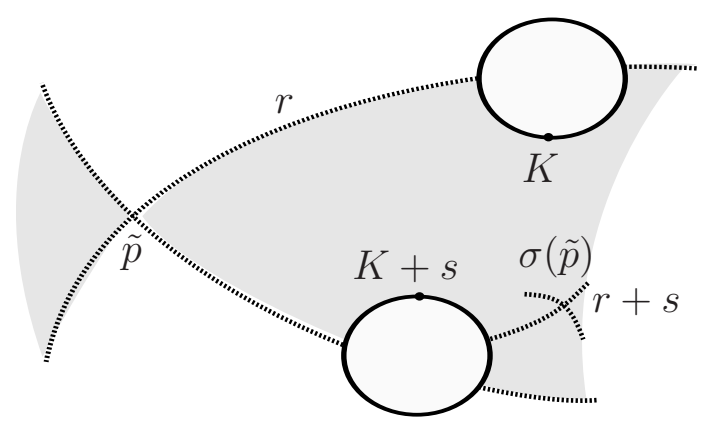

Figure 5.4: A singular level crossing boundary components

At this point we have a good picture of what happens in this case, and it turns out that we have a globally solvable system.

Construct a covering space $\widetilde{M_{1}}$ with boundary for $M_{1}$, and a covering space $\widetilde{M}_{2}$ with boundary for $M_{2}$.

If the other saddle point, $q$, is in $M_{2}$, we proceed as follows. The noosing of last lemma gives us leaves in $M_{1}$, which we call $F_{p}^{i}$, with $i=1,2$, containing separatrices of $p$, and leaves in $M_{2}$, which we call $F_{q}^{i}$, with $i=1,2$, containing separatrices of $q$. Such leaves split $\alpha$ in four pieces: a path which, together with $F_{p}^{1}, F_{p}^{2}$ and $p$, bounds a closed disk $D_{1}$; a path which, together with $F_{q}^{1}, F_{q}^{2}$ and $q$, bounds a closed disk $D_{2}$; a path $\zeta_{1}$ which connects, say, $F_{p}^{1}$ to $F_{q}^{1}$; and a path $\zeta_{2}$ which connects $F_{p}^{2}$ to $F_{q}^{2}$. Each closed disk contains a different critical point of $\left.B\right|_{\alpha}$.

Notice that the simple closed curve on $M$ consisting of the singular points and $F_{p}^{1}, F_{p}^{2}, F_{q}^{1}, F_{q}^{2}, \zeta_{1}, \zeta_{2}$ is in the collar. By natural approximations of the leaves in this curve, we construct in the collar of $M$ an almost transversal curve that contains the saddles. Therefore, Remark 4.4 says that $b$ is obtained from Morse forms on tori 
without singular points by means of Case C. The global solvability now follows from Corollary 3.16 .

If $p$ and $q$ are both in $M_{1}$, but the path obtained when we noose $p$ does not contain $q$, we proceed similarly. The noosing of last lemma gives us leaves in $M_{1}$, which we call $F_{p}^{i}$, with $i=1,2$, containing separatrices of $p$, and $F_{q}^{i}$, with $i=1,2$, containing separatrices of $q$, and again we can construct in the collar of $M$ an almost transversal that contains the saddles.

Finally, there is a possibility that the arc obtained when we noose $p$ contains $q$ (see Figure 5.5). The projection of this arc contains the compactifiable leaf connecting $p$ and $q$, and, together with a piece of $\alpha$, bounds a closed disk $D_{1}$. This closed disk contains a critical point of $\left.B\right|_{\alpha}$.

By repeating the arguments of Lemma 5.3, there is another arc in the covering $\widetilde{M}_{1}$ noosing the saddles. The projection of this arc, together with a piece of $\alpha$, bounds a closed disk $D_{2}$ containing the other critical point of $\left.B\right|_{\alpha}$. However, the projection of this arc also contains the compactifiable leaf.

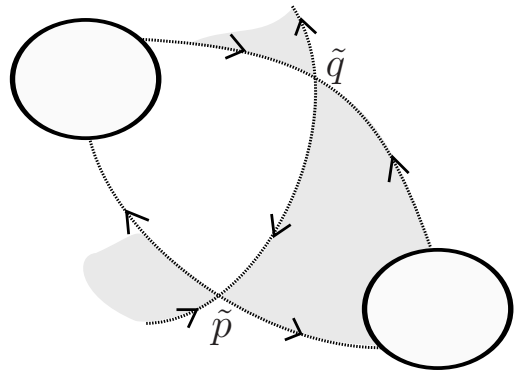

Figure 5.5: Saddles connected by a leaf in $M_{1}$

We then consider $\widetilde{M_{1}^{*}} \doteq \widetilde{M_{1}} \backslash\left(\operatorname{int}\left(D_{1}\right) \cup \operatorname{int}\left(D_{2}\right)\right)$ (notice that this set covers a torus $M_{1}^{*}$ having a singular curve in the boundary). As a result, it holds

Claim. The sublevels and the superlevels of $\left.B\right|_{\widetilde{M_{1}^{*}}}$ are connected.

Proof. First notice that in the plane or in the cylinder each superlevel and each sublevel of $B$ has only one unbounded component, hence the same must occur with $\left.B\right|_{\widetilde{M_{1}^{*}}}$.

Now, $\left.B\right|_{\widetilde{M_{1}^{*}}}$ does not have bounded components of superlevels or sublevels since we have removed the candidates to be points of local maximum or local minimum.

In order to build a solution for $M$, we need $M_{2}^{*}=M_{2} \cup D_{1} \cup D_{2}$ to be covered by $\widetilde{M_{2}^{*}}$ such that the sublevels and the superlevels of $\left.B\right|_{\widetilde{M_{2}^{*}}}$ are connected too. But the torus $M_{2}$ can be viewed as a torus where a Morse form without singular points is defined and to which a pipe as in Case $\mathrm{C}$ was attached. Therefore, it is covered by a surface $\widetilde{M}_{2}$ with boundary such that $\left.B\right|_{\widetilde{M_{2}}}$ has connected sublevels and superlevels, and the same occurs with $M_{2}^{*}$. 
To $M_{1}^{*}$ and $M_{2}^{*}$ we apply Lemma 3.7 (or 3.10) and Propositions 3.8 and 3.9 (or 3.11 and 3.12). Afterwards, the global solvability comes from the gluing as in Proposition 3.14 .

Example 5.4. Consider the function $B(x, y)=c_{1} x+d_{1} y$ defined on the plane, with $c_{1}, d_{1}$ linearly independent over $\mathbb{Q}$. In a fundamental square, we perturb the levels sets by creating saddles and points of local maximum and local minimum, as shown in Figure 5.6, and in such a way that a saddle is connected to a translate of the other saddle. Choose open sets as depicted below so the foliation there assembles with the foliation of a torus on which $\omega_{2}=c_{2} d \theta+d_{2} d \phi$ is defined, with $c_{1}, d_{1}, c_{2}, d_{2}$ being linearly independent over $\mathbb{Q}$. We can carry out the connected sum as in Case $C$ so that the resulting bitorus has only one compactifiable arc, which is connecting the saddles.

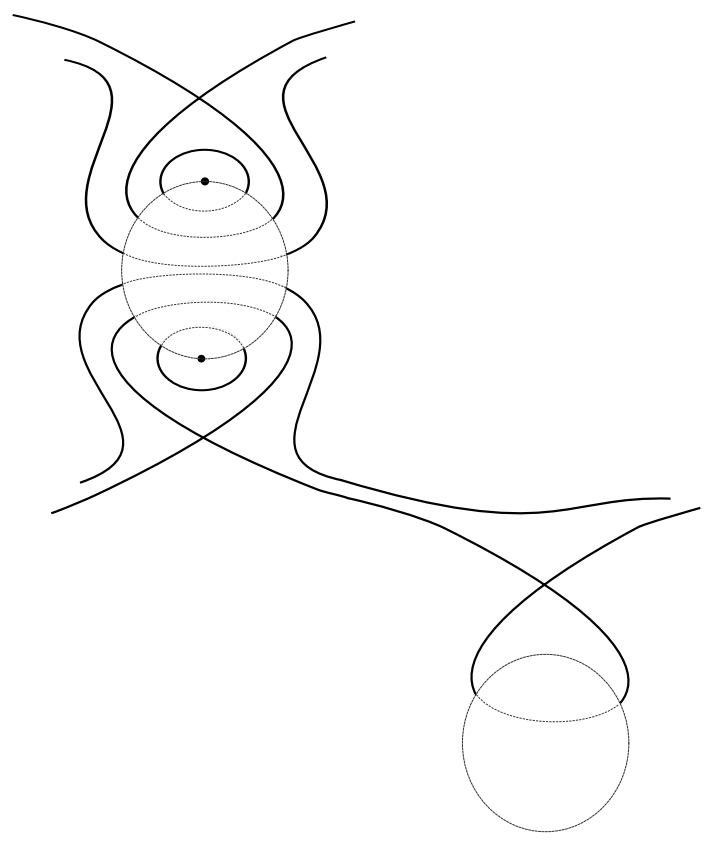

Figure 5.6: An example having only one compactifiable arc

Example 5.5 (A real analytic foliation). Intuitively, this example is a perturbation of the previous one, this time with only one critical point. See Figure 5.7.

With the above notation, we have a real analytic function $B$ in $\widetilde{M}_{1}$ such that there is a sequence of Morse functions $\left\{B_{n}\right\}_{n \in \mathbb{N}}$ defined on $\widetilde{M}_{1}$ (we keep the boundary components fixed) and uniformly convergent to $B$. We can assume that $B_{n}$ has the same configuration of the previous example and is equal to $B$ in a neighborhood of the fixed boundary components, for every $n \in \mathbb{N}$. Hence, the superlevels and the sublevels of $B$ are connected in $\widetilde{M_{1}^{*}}$, and we can produce an example of a globally solvable system for the bitorus with a real analytic 1-form.

The next statement sums up the results of this chapter and Theorem 4.8 for the bitorus. 


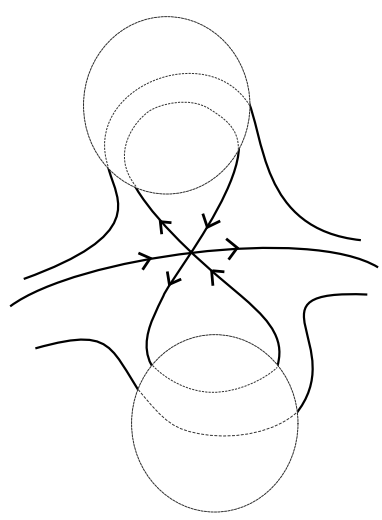

Figure 5.7: A real analytic example

Theorem 5.6. Suppose b is a Morse 1-form defined on the bitorus. If b has only saddles as critical points, or equivalently, there are no components of sublevels or superlevels in the universal covering $D$, then the operator $\mathbb{L}$ is globally solvable.

By using Theorem 2.1 together with Proposition 2.2 (ii), we have

Theorem 5.7. Suppose $b$ is a Morse 1-form defined on the bitorus. Then the operator $\mathbb{L}$ is globally solvable if and only if there are no components of sublevels or superlevels in $D$ where a primitive of $b$ is bounded. 


\section{CHAPTER 6}

FURTHER EXAMPLES

\subsection{A case with rank 1}

Now we deal with a real closed non-exact 1 -form $b$ on a surface of genus $g$, as in Example $(i)$, Section 1.3. Recall that $\widetilde{M}$ is embedded in $\mathbb{R}^{3}$ (see Figure 6.1). We consider the translation $\tau$ as a restriction of the translation on $\mathbb{R}^{3}$ defined by $t \mapsto t+(0,2 \pi)$, with $(0,2 \pi) \in \mathbb{R}^{2} \times \mathbb{R}$.

Because of the stronger hypothesis about the rank, we will be allowed to require only that $b$ is a smooth 1 -form instead of Morse.

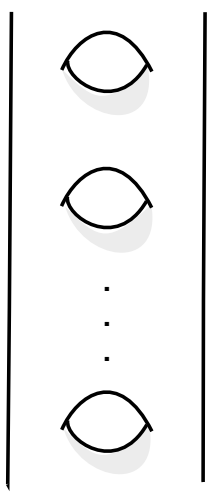

Figure 6.1: Covering space for rank 1

In order to prove the global solvability, our assumption will be that in $\widetilde{M}$ there are no components of sublevels or superlevels on which $B$ is bounded.

As a matter of fact, in this example the sublevels and the superlevels have only one unbounded component in $\widetilde{M}$. Hence, the non-connectedness of a given superlevel is equivalent to the existence of a bounded component of this superlevel, thus, a component of this superlevel where $B$ is bounded (and analogously for the sublevels). 
Therefore, we can suppose that the sublevels and the superlevels of $B$ are connected in $\widetilde{M}$.

We will proceed like in Section 3.3.2. Recall that $B(t+(0,2 \pi))=B(t)+d^{\prime}$, with $d^{\prime}$ non-zero and $t \in \widetilde{M}$. Consider the set $S=\left\{\tau=\left(\tau_{1}, \tau_{2}\right) \in \mathbb{R}^{2} \times \mathbb{R}:\left|\tau_{2}\right| \leqslant \nu\right\}$, a horizontal strip containing all the levels of the points in a fundamental domain.

Set, as before, $\Omega_{r}=\{\tau \in \widetilde{M}: B(\tau)<r\}$ and $\Omega^{r}=\{\tau \in \widetilde{M}: B(\tau)>r\}$. We have

Lemma 6.1. The sets $K_{r}=\Omega_{r} \cap S$ and $K^{r}=\Omega^{r} \cap S$ are bounded and path-connected.

As before, one can compute the Fourier coefficients of the candidate to the problem's solution on $\widetilde{M}$, by solving a differential equation for each $\xi \in \mathbb{Z}$, which yields

$$
\widehat{u}(t, \xi)=\int_{t_{0}}^{t} v+K_{\xi} e^{\xi B(t)},
$$

where $v(s)=e^{-\xi[B(s)-B(t)]} \hat{f}(s, \xi)$.

If we impose the periodicity in order to define a solution on $M$, we determine the coefficients of such a solution, namely

$$
\widehat{u}(t, \xi)=\rho(\xi) \int_{t}^{t+(0,2 \pi)} v,
$$

where $\rho(\xi)=\left(e^{-\xi d^{\prime}}-1\right)^{-1}$.

Again, we also have

$$
\widehat{u}(t, \xi)=\rho(-\xi) \int_{t}^{t-(0,2 \pi)} v
$$

The paths that we look for in order to prove the decay of the coefficients are given by the next propositions.

Proposition 6.2. For each $\xi \in \mathbb{Z}$ and $t$ in a fundamental domain there exist paths $\gamma(t, \xi)$ in $\widetilde{M}$, connecting $t$ to $t+(0,2 \pi)$ or $t-(0,2 \pi)$, with the following properties:

(i) $B(\tau) \leqslant B(t)+\frac{1}{1+|\xi|}, \quad \tau \in \gamma(t, \xi)$

(ii) $|\gamma(t, \xi)| \leqslant C(1+|\xi|)$.

Proposition 6.3. For each $\xi \in \mathbb{Z}$ and $t$ in a fundamental domain there exist paths $\gamma(t, \xi)$ in $\widetilde{M}$, connecting $t$ to $t+(0,2 \pi)$ or $t-(0,2 \pi)$, with the following properties: 
(i) $B(\tau) \geqslant B(t)-\frac{1}{1+|\xi|}, \quad \tau \in \gamma(t, \xi)$

(ii) $|\gamma(t, \xi)| \leqslant C(1+|\xi|)$.

In short, using Theorem 2.1 together with Proposition 2.2 (ii), we have proved in this section the following equivalence

Theorem 6.4. Under the above assumptions on $b$, the operator $\mathbb{L}$ is globally solvable if and only if there are no components of sublevels and superlevels in $D$ where $B$ is bounded.

\subsection{Non-solvability revisited}

It is interesting to give some examples of constructions of 1-forms on the bitorus in which we start from one or two tori such that at least in one of them the 1-form defines a non-globally solvable system, and yet the final result is a globally solvable system.

Example 6.5. Consider the height function $H$ on a torus $T$ embedded in $\mathbb{R}^{3}$ with coordinates $(x, y, z)$ and standing vertically on the plane $x y . B y[\mathrm{CH}]$, the system defined by the 1 -form $h=d H$ is globally solvable.

Take two sufficiently small closed disks $D_{1} \subset D_{2}$ containing the point of maximal of $H$. We define a cutoff function $\varphi$ equal to 0 in $D_{1}$ and equal to 1 in $T \backslash \operatorname{int}\left(D_{2}\right)$. Then, by $[\mathrm{CH}]$, the system defined by the 1-form $h=d(\varphi H)$ is not globally solvable.

Define now a closed Morse 1-form b on a bitorus as follows. Cut two sufficiently small open disks $\mathrm{O}_{1}$ and $\mathrm{O}_{2}$ such that $O_{1}$ contains the point of minimum of $H$, and $D_{2} \subset O_{2}$. On the surface as in Figure 6.1 a smooth pseudoperiodic function $B$ is defined satisfying $B\left(\tau^{n}(t)\right)=H(t)+n . a$, for $t \in T \backslash\left(O_{1} \cup O_{2}\right)$ and $a \neq 0$. Hence, we set $b \doteq d B$.

This 1-form is defined on a bitorus and has only one non-zero period. In the covering space there are only connected sublevels and superlevels, thus the system is globally solvable.

Example 6.6. In the same spirit, it is possible to view the construction presented in Case B, Chapter 3, as the connected sum of $T_{1}$ and $T_{2}^{\prime}$, where $T_{2}^{\prime}$ is such that on it $\omega_{2}^{\prime}$ is defined by extending $\left.b\right|_{M_{2}}$ (see Remark 5.1).

As we will have a component of a sublevel (or a superlevel) on which a local primitive of $\omega_{2}^{\prime}$ is bounded, the system on $T_{2}^{\prime}$ is not globally solvable, but we have proved that the system defined by $b$ on the bitorus can be globally solvable.

Conversely, in Example 2.4 we have constructed a non-globally solvable system on the bitorus from 1-forms defining globally solvable systems on two tori. 
[Ar] Arnold, V. I., Topological and Ergodic Properties of Closed 1-forms with Incommensurable Periods, Funct. Anal. Appl. 25 (2) (1991), 81-90.

[BCH] Berhanu, S.; Cordaro, P.; Hounie, J., An Introduction to Involutive Structures, Cambridge University Press, 2008.

[BCM] Bergamasco, A.; Cordaro, P.; Malagutti, P., Global Hypoelliptic Systems of Vector Fields, J. Funct. Anal. 114 (1993), 267-285.

[BK] Bergamasco, A.; Kirilov, A., Global Solvability for a Class of Overdetermined Systems, J. Funct. Anal. 252 (2007), no. 2, 603-629.

[BKNZ] Bergamasco, A.; Kirilov, A.; Nunes, W.; Zani, S., On the Global Solvability for Overdetermined Systems, Trans. Amer. Math. Soc. 364 (2012), no. 9, 4533-4549.

[Ca] Camacho, C.; Lins Neto, A., Geometric Theory of Foliations, Birkhäuser Boston, Inc., 1985.

[CH] Cardoso, F.; Hounie, J., Global Solvability of an Abstract Complex, Proc. Amer. Math. Soc. 65 (1977), 117-124.

[Far] Farber, M., Topology of Closed One-Forms, Math. Surv., no. 108, AMS, 2004.

[Fe] Fenn, R., What is the Geometry of a Surface?, Amer. Math. Mon. 90 (1983), no.2, $87-98$.

[Gel] Gelbukh, I., On the Structure of a Morse Form Foliation, Czechoslovak Math. J. 59 (134) (2009), no. 1, 207-220.

[Ha] Hatcher, A., Algebraic Topology, Cambridge University Press, 2002.

[Hae] Haefliger, A., Variétés Feuillitées, Ann. Scuola Norm. Sup. Pisa 16 (1962), 367397. 
[Im1] Imanishi, H., On Codimension One Foliations Defined by Closed One Forms with Singularities, J. Math. Kyoto Univ. 19 (1979), no. 2, 285-291.

[Im2] Imanishi, H., Structure of Codimension 1 Foliations without Holonomy on Manifolds with Abelian Fundamental Group, J. Math. Kyoto Univ. 19 (1979), no. 3, 481-495.

[Jo] Jost, J., Compact Riemann Surfaces, Springer-Verlag, Berlin, 2006.

[Lev] Levitt, G., Pantalons et Feuilletages des Surfaces, Topology, vol. 21 (1982), no. 1, 9-33.

[Tr1] Treves, F., Topological Vector Spaces, Distributions and Kernels, Academic Press, New York, 1967.

[Tr2] Treves, F., Study of a Model in the Theory of Complexes of Pseudodifferential Operators, Ann. of Math. (2) 104 (1976), 269-324. 


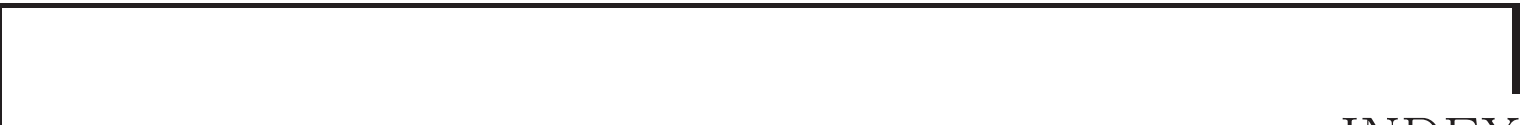

Almost transversal curve, 37

Attaching

handles, 23

pipes, 23

Characteristic set, 2

Collar, 3

Compatibility conditions, 7

Component

maximal, 21

minimal, 21

Connected sum, 22

Covering space

Minimal, 5

Normal, 5

Foliation, 19

orientable, 20

Fundamental domain, 4, 6

Generic form, 41

Globally solvable operator, 8

Homology group, 3

Homotopy group, 3, 5

Leaf, 19

compact, 21

compactifiable, 21

non-compactifiable, 21

Levi form, 2

Lollipops, 7, 46, 49
Morse form, 19

Noosing the saddles, 39

Periodic manifold, 6

Periods, 3

Pipe, 23

Pseudoperiodic function, 5

Rank, 3

Rapid decay, 2

Separatrix, 21

Theorem

Levitt's, 37

Stokes', 3

Transversal lines, 27 